\title{
OPERATIONAL BEHAVIOR OF THE MAP/G/1 QUEUE UNDER N-POLICY WITH A SINGLE VACATION AND SET-UP ${ }^{1}$
}

\author{
HO WOO LEE \\ Sung Kyun Kwan University \\ Department of Systems Management Engineering \\ Su Won, Korea 440-746 \\ E-mail:hwlee@yurim.skku.ac.kr \\ BOO YONG AHN \\ $S K C \& C$ \\ Seoul, Korea
}

(Received April, 2000; Revised June, 2001)

\begin{abstract}
This paper considers the MAP/G/1 queue under $N$-policy with a single vacation and set-up. We derive the vector generating functions of the queue length at an arbitrary time and at departures in decomposed forms. We also derive the Laplace-Stieltjes transform of the waiting time. Computation algorithms for mean performance measures are provided.
\end{abstract}

Key words: MAP/G/1, Queue Length, Decomposition.

AMS subject classifications: 60K25, 90B22, 68M20.

\section{Introduction}

We consider the MAP/G/1 queue under $N$-policy with a single vacation and set-up. As soon as there are no customers to serve, the server takes a vacation (vacation period). After the vacation, if the server finds $N$ or more customers, it starts a set-up of a random length (set-up period). If not, the server stays in the system until $N$ customers accumulate (dormant period). After the set-up, the server continuously serves the customers until the system empties (buy period). Thus, a cycle consists of a vacation period, a dormant period, a set-up period and a busy period. The service, vacation and set-up times are assumed to be mutually independent with general distributions. We also assume that they are independent of the phase of the underlying Markov chain. Customers arrive according to the Markovian Arrival Process (MAP) with parameter matrices $\boldsymbol{C}$ and $\boldsymbol{D}$. For the formal definition of MAP, readers are advised to see Lucantoni, et al. [10].

We derive the vector generating functions of the queue length at an arbitrary time and at departures in decomposed forms. We also derive the Laplace-Stieltjes transform of the waiting time. Computation algorithms for mean performance measures are provided.

\footnotetext{
${ }^{1}$ This work was supported in part by KOSEF through Statistical Research Center for Complex Systems at Seoul National University.
} 
Lee et al. [5] studied the MAP/G/1 system under multiple and single vacations with $N$ policy and showed that the vector generating functions of the queue length at an arbitrary time and at departures are decomposed into two parts: one is the vector generating function of the queue length at an arbitrary idle time, and the other is an unidentified matrix generating function. The authors [6] also confirmed that those decompositions hold for the MAP/G/1 system under $N$-policy with multiple vacations and set-up. We show that those decompositions also hold for this system with a single vacation and set-up.

Kasahara et al. [2] studied the MAP/G/1 queue with $N$-policy and multiple vacations. They used the matrix-analytic approach pioneered by Neuts [11, 12], and his colleagues [8$10,15,16]$. In this paper, we employ the method of supplementary variables. This method directly provides us with the queue length distributions at an arbitrary time. Departure point queue length and virtual waiting time distributions can be obtained as by-products. For works in this category, see [1, 3-6, 13, 14].

The remainder of the paper is organized as follows. In Section 2, we develop the vector system equations and solve the equations by using the eigenvalues and eigenvectors of $-(\boldsymbol{C}+z \boldsymbol{D})$. We derive the vector generating functions of the queue length at an arbitrary time and at departures in decomposed forms. Interpretations of the decompositions are presented. In Section 3, we derive the Laplace-Stieltjes transform of the waiting time of an arbitrary customer. In Section 4, we present the computation algorithms for mean performance measures. We consider some special cases in Section 5.

\section{The Queue Length}

We first develop the system equations. Let us define the following notations:

$N$ : steady-state queue length (number of customers in the system, including the one in service)

$J: \quad$ the phase of the underlying Markov chain (UMC)

$S_{R}, V_{R}, H_{R}$ : remaining service time, vacation time and set-up time

$\boldsymbol{C}, \boldsymbol{D}:(m \times m)$ parameter matrices of the Markovian arrival process

$\boldsymbol{C}_{i j}, \boldsymbol{D}_{i j}$ : $\quad(i, j)$-element of $\boldsymbol{C}, \boldsymbol{D}$

$\pi_{i}=\operatorname{Pr}[J=i],(1 \leq i \leq m)$

$\boldsymbol{\pi}=\left(\pi_{1}, \pi_{2}, \ldots, \pi_{m}\right)$

$\lambda=\boldsymbol{\pi} \boldsymbol{D} \boldsymbol{e}$ : mean arrival rate

$\rho=\lambda E(S): \quad$ traffic intensity

$\zeta(t)= \begin{cases}1, & \text { (server is on a vacation) } \\ 2, & \text { (server is in a dormant period) } \\ 3, & \text { (server is in a set-up) } \\ 4, & \text { (server is busy) }\end{cases}$

Let us define the following probabilities:

$$
\begin{gathered}
p_{n, i}^{(V)}(x) d x=\operatorname{Pr}\left[\zeta=1, N=n, J=i, V_{R} \in(x, x+d x]\right], \quad(n \geq 0,1 \leq i \leq m) \\
p_{n, i}^{(D)}=\operatorname{Pr}[\zeta=2, N=n, J=i], \quad(0 \leq n \leq N-1,1 \leq i \leq m) \\
p_{n, i}^{(S)}(x) d x=\operatorname{Pr}\left[\zeta=3, N=n, J=i, V_{R} \in(x, x+d x]\right], \quad(n \geq N, 1 \leq i \leq m) \\
p_{n, i}^{(B)}(x) d x=\operatorname{Pr}\left[\zeta=4, N=n, J=i, S_{R} \in(x, x+d x]\right], \quad(n \geq 1,1 \leq i \leq m) .
\end{gathered}
$$


Then, defining $s(x), v(x)$ and $h(x)$ as the pdf of the service time, vacation time and set-up time respectively, the above probabilities satisfy the following equations:

$$
\begin{aligned}
& 0=\sum_{j=1}^{m} p_{0, j}^{(D)} \boldsymbol{C}_{j i}+p_{0, i}^{(V)}(0),(1 \leq i \leq m) \\
& 0=\sum_{j=1}^{m} p_{n, j}^{(D)} \boldsymbol{C}_{j i}+\sum_{j=1}^{m} p_{n-1, j}^{(D)} \boldsymbol{D}_{j i}+p_{n, i}^{(V)}(0),(1 \leq n \leq N-1,1 \leq i \leq m) \\
& -\frac{d}{d x} p_{1, i}^{(B)}(x)=\sum_{j=1}^{m} p_{1, j}^{(B)}(x) \boldsymbol{C}_{j i}+p_{2, i}^{(B)}(0) s(x),(1 \leq i \leq m) \\
& -\frac{d}{d x} p_{k, i}^{(B)}(x)=\sum_{j=1}^{m} p_{k, j}^{(B)}(x) \boldsymbol{C}_{j i}+p_{k+1, i}^{(B)}(0) s(x)+\sum_{j=1}^{m} p_{k-1, j}^{(B)}(x) \boldsymbol{D}_{j i}, \\
& (2 \leq k \leq N-1,1 \leq i \leq m) \\
& -\frac{d}{d x} p_{N, i}^{(B)}(x)=\sum_{j=1}^{m} p_{N, j}^{(B)}(x) \boldsymbol{C}_{j i}+p_{N+1, i}^{(B)}(0) s(x)+\sum_{j=1}^{m} p_{N-1, j}^{(B)}(x) \boldsymbol{D}_{j i}+p_{N, i}^{(S)}(0) s(x), \\
& (1 \leq i \leq m) \\
& -\frac{d}{d x} p_{n, i}^{(B)}(x)=\sum_{j=1}^{m} p_{n, j}^{(B)}(x) \boldsymbol{C}_{j i}+p_{n+1, i}^{(B)}(0) s(x)+\sum_{j=1}^{m} p_{n-1, j}^{(B)}(x) \boldsymbol{D}_{j i}+p_{n, i}^{(S)}(0) s(x), \\
& (n \geq N+1,1 \leq i \leq m) \\
& -\frac{d}{d x} p_{0, i}^{(V)}(x)=\sum_{j=1}^{m} p_{0, j}^{(V)}(x) \boldsymbol{C}_{j i}+p_{1, i}^{(B)}(0) v(x), \quad(1 \leq i \leq m) \\
& -\frac{d}{d x} p_{n, i}^{(V)}(x)=\sum_{j=1}^{m} p_{n, j}^{(V)}(x) \boldsymbol{C}_{j i}+\sum_{j=1}^{m} p_{n-1, j}^{(V)}(x) \boldsymbol{D}_{j i},(n \geq 1,1 \leq i \leq m) \\
& -\frac{d}{d x} p_{N, i}^{(S)}(x)=\sum_{j=1}^{m} p_{N, j}^{(S)}(x) \boldsymbol{C}_{j i}+\sum_{j=1}^{m} p_{N-1, j}^{(D)} \boldsymbol{D}_{j i} h(x)+p_{N}^{(V)}(0) h(x),(1 \leq i \leq m) \\
& -\frac{d}{d x} p_{n, i}^{(S)}(x)=\sum_{j=1}^{m} p_{n, j}^{(D)}(x) \boldsymbol{C}_{j i}+\sum_{j=1}^{m} p_{n-1, j}^{(S)} \boldsymbol{D}_{j i}+p_{n}^{(V)}(0) h(x),(n \geq N+1,1 \leq i \leq m) .
\end{aligned}
$$

Let us define the $(1 \times m)$ row vectors as follows:

$$
\begin{gathered}
\boldsymbol{p}_{n}^{(D)}=\left(p_{n, 1}^{(D)}, p_{n, 2}^{(D)}, \ldots, p_{n, m}^{(D)}\right), \boldsymbol{p}_{n}^{(B)}(x)=\left(p_{n, 1}^{(B)}(x), p_{n, 2}^{(B)}(x), \ldots, p_{n, m}^{(B)}(x)\right) \\
\boldsymbol{p}_{n}^{(V)}(x)=\left(p_{n, 1}^{(V)}(x), p_{n, 2}^{(V)}(x), \ldots, p_{n, m}^{(V)}(x)\right), \boldsymbol{p}_{n}^{(S)}(x)=\left(p_{n, 1}^{(S)}(x), p_{n, 2}^{(S)}(x), \ldots, p_{n, m}^{(S)}(x)\right) .
\end{gathered}
$$

Then, the above system equations can be converted to the vector system equations as follows:

$$
\mathbf{0}=\boldsymbol{p}_{0}^{(D)} \boldsymbol{C}+\boldsymbol{p}_{0}^{(V)}(0)
$$




$$
\begin{gathered}
\mathbf{0}=\boldsymbol{p}_{n}^{(D)} \boldsymbol{C}+\boldsymbol{p}_{n-1}^{(D)} \boldsymbol{D}+\boldsymbol{p}_{n}^{(V)}(0),(1 \leq n \leq N-1) \\
-\frac{d}{d x} \boldsymbol{p}_{1}^{(B)}(x)=\boldsymbol{p}_{1}^{(B)}(x) \boldsymbol{C}+\boldsymbol{p}_{2}^{(B)}(0) s(x) \\
-\frac{d}{d x} \boldsymbol{p}_{n}^{(B)}(x)=\boldsymbol{p}_{n}^{(B)}(x) \boldsymbol{C}+\boldsymbol{p}_{n+1}^{(B)}(0) s(x)+\boldsymbol{p}_{n-1}^{(B)}(x) \boldsymbol{D},(2 \leq n \leq N-1) \\
-\frac{d}{d x} \boldsymbol{p}_{N}^{(B)}(x)=\boldsymbol{p}_{N}^{(B)}(x) \boldsymbol{C}+\boldsymbol{p}_{N+1}^{(B)}(0) s(x)+\boldsymbol{p}_{N-1}^{(B)}(x) \boldsymbol{D}+\boldsymbol{p}_{N}^{(S)}(0) s(x) \\
-\frac{d}{d x} \boldsymbol{p}_{n}^{(B)}(x)=\boldsymbol{p}_{n}^{(B)}(x) \boldsymbol{C}+\boldsymbol{p}_{n+1}^{(B)}(0) s(x)+\boldsymbol{p}_{n-1}^{(B)}(x) \boldsymbol{D}+\boldsymbol{p}_{n}^{(S)}(0) s(x), \\
-\frac{d}{d x} \boldsymbol{p}_{0}^{(V)}(x)=\boldsymbol{p}_{0}^{(V)}(x) \boldsymbol{C}+\boldsymbol{p}_{1}^{(B)}(0) v(x) \\
-\frac{d}{d x} \boldsymbol{p}_{n}^{(V)}(x)=\boldsymbol{p}_{n}^{(V)}(x) \boldsymbol{C}+\boldsymbol{p}_{n-1}^{(V)} \boldsymbol{D},(n \geq 1) \\
-\frac{d}{d x} \boldsymbol{p}_{N}^{(S)}(x)=\boldsymbol{p}_{N}^{(S)}(x) \boldsymbol{C}+\boldsymbol{p}_{N-1}^{(D)} \boldsymbol{D h}(x)+\boldsymbol{p}_{N}^{(V)}(0) h(x) \\
-\frac{d}{d x} \boldsymbol{p}_{n}^{(S)}(x)=\boldsymbol{p}_{n}^{(S)}(x) \boldsymbol{C}+\boldsymbol{p}_{n-1}^{(S)}(x) \boldsymbol{D}+\boldsymbol{p}_{n}^{(V)}(0) h(x),(n \geq N+1) .
\end{gathered}
$$

Let us define the following vector generating functions

$$
\begin{gathered}
\boldsymbol{p}^{(D)}(z)=\sum_{n=0}^{N-1} \boldsymbol{p}_{n}^{(D)} z^{n}, \boldsymbol{p}^{(B)}(z, x)=\sum_{n=1}^{\infty} \boldsymbol{p}_{n}^{(B)}(x) z^{n}, \boldsymbol{p}^{(V)}(z, x)=\sum_{n=0}^{\infty} \boldsymbol{p}_{n}^{(V)}(x) z^{n} \\
\boldsymbol{p}^{(S)}(z, x)=\sum_{n=N}^{\infty} \boldsymbol{p}_{k}^{(S)}(x) z^{k}
\end{gathered}
$$

Multiplying (2.3)-(2.6) by $z^{n}$ and summing over $n$, yields

$$
\begin{aligned}
-\frac{d}{d x} \boldsymbol{p}^{(B)}(z, x) & =\boldsymbol{p}^{(B)}(z, x)(\boldsymbol{C}+z \boldsymbol{D}) \\
& +\left[\frac{1}{z} \boldsymbol{p}^{(B)}(z, 0)-\boldsymbol{p}_{1}^{(B)}(0)+\boldsymbol{p}^{(S)}(z, 0)\right] s(x) .
\end{aligned}
$$

Similarly, from (2.7) and (2.8), we get

$$
-\frac{d}{d x} \boldsymbol{p}^{(V)}(z, x)=\boldsymbol{p}^{(V)}(z, x)(\boldsymbol{C}+z \boldsymbol{D})+\boldsymbol{p}_{1}^{(B)}(0) v(x) .
$$

From (2.9) and (2.10), we get

$$
\begin{aligned}
-\frac{d}{d x} \boldsymbol{p}^{(S)}(z, x) & =\boldsymbol{p}^{(S)}(z, x)(\boldsymbol{C}+z \boldsymbol{D}) \\
& +\left[z^{N} \boldsymbol{p}_{N-1}^{(D)} \boldsymbol{D}+\sum_{n=N}^{\infty} \boldsymbol{p}_{n}^{(V)}(0) z^{n}\right] h(x) .
\end{aligned}
$$

Let us define the Laplace transform as follows: 


$$
\begin{gathered}
\boldsymbol{p}^{*(B)}(z, \theta)=\int_{0}^{\infty} e^{-\theta x} \boldsymbol{p}^{(B)}(z, x) d x, \boldsymbol{p}^{*(V)}(z, \theta)=\int_{0}^{\infty} e^{-\theta x} \boldsymbol{p}^{(V)}(z, x) d x, \\
\boldsymbol{p}^{*(S)}(z, \theta)=\int_{0}^{\infty} e^{-\theta x} \boldsymbol{p}^{(S)}(z, x) d x .
\end{gathered}
$$

Taking the Laplace transforms of both sides of (2.11)-(2.13), we obtain

$$
\begin{aligned}
\boldsymbol{p}^{*(B)}(z, \theta)(\theta \boldsymbol{I}+\boldsymbol{C}+z \boldsymbol{D}) & =\left[1-\frac{S^{*}(\theta)}{z}\right] \boldsymbol{p}^{(B)}(z, 0) \\
& -\left[\boldsymbol{p}^{(S)}(z, 0)-\boldsymbol{p}_{1}^{(B)}(0)\right] S^{*}(\theta) \\
\boldsymbol{p}^{*(V)}(z, \theta)(\theta \boldsymbol{I}+\boldsymbol{C}+z \boldsymbol{D}) & =\boldsymbol{p}^{(V)}(z, 0)-\boldsymbol{p}_{1}^{(B)}(0) V^{*}(\theta) \\
\boldsymbol{p}^{*(S)}(z, \theta)(\theta \boldsymbol{I}+\boldsymbol{C}+z \boldsymbol{D}) & =\boldsymbol{p}^{(S)}(z, 0) \\
& -\left[\sum_{n=N}^{\infty} \boldsymbol{p}_{n}^{(V)}(0) z^{n}+z^{N} \boldsymbol{p}_{N-1}^{(D)} \boldsymbol{D}\right] H^{*}(\theta) .
\end{aligned}
$$

From (2.1) and (2.2), we get

$$
z^{N} \boldsymbol{p}_{N-1}^{(D)} \boldsymbol{D}=\sum_{n=0}^{N-1} \boldsymbol{p}_{n}^{(V)}(0) z^{n}+\boldsymbol{p}^{(D)}(z)(\boldsymbol{C}+z \boldsymbol{D})
$$

Using (2.17) in (2.16), we get

$$
\begin{aligned}
\boldsymbol{p}^{*(S)}(z, \theta)(\theta \boldsymbol{I}+\boldsymbol{C}+z \boldsymbol{D}) & =\boldsymbol{p}^{(S)}(z, 0) \\
& -\left[\boldsymbol{p}^{(D)}(z)(\boldsymbol{C}+z \boldsymbol{D})+\boldsymbol{p}^{(V)}(z, 0)\right] H^{*}(\theta) .
\end{aligned}
$$

Now, we obtain $\boldsymbol{p}^{(B)}(z, 0), \boldsymbol{p}^{(V)}(z, 0)$ and $\boldsymbol{p}^{(S)}(z, 0)$ contained in (2.14), (2.15) and (2.18). They concern the queue length and the phase of the underlying Markov chain (UMC) at the ending points of a busy period, a vacation, and a set-up. This can be accomplished by eliminating the left-hand side of (2.14), (2.15) and (2.18). For this purpose, we use the eigenvalues of the matrix $-(\boldsymbol{C}+z \boldsymbol{D})$ and their right eigenvectors. Let $\alpha_{i}(z)$, $(i=1,2, \ldots, m)$ be the eigenvalues. Let $\xi_{i}(z)$ be the right eigenvector of $\alpha_{i}(z)$. It is wellknown that $\alpha_{i}(z)$ and $\xi_{i}(z)$ are related by (see Strang [17], for example)

$$
-(\boldsymbol{C}+z \boldsymbol{D}) \xi_{i}(z)=\alpha_{i}(z) \xi_{i}(z)
$$

or

$$
\left[\alpha_{i}(z) \boldsymbol{I}+\boldsymbol{C}+z \boldsymbol{D}\right] \xi_{i}(z)=\mathbf{0}, \quad(1 \leq i \leq m)
$$

Using $\alpha_{i}(z)$ in $\theta$ of (2.15) and postmultiplying both sides by $\xi_{i}(z)$, we get

$$
\begin{aligned}
& \boldsymbol{p}^{*(V)}\left(z, \alpha_{i}(z)\right)\left[\alpha_{i}(z) \boldsymbol{I}+\boldsymbol{C}+z \boldsymbol{D}\right] \xi_{i}(z) \\
& =\left[\boldsymbol{p}^{(V)}(z, 0)-\boldsymbol{p}_{1}^{(B)}(0) V^{*}\left(\alpha_{i}(z)\right)\right] \xi_{i}(z) .
\end{aligned}
$$

Then, the left-hand side of (2.19a) vanishes and we get 


$$
\boldsymbol{p}^{(V)}(z, 0) \xi_{i}(z)=\boldsymbol{p}_{1}^{(B)}(0) V^{*}\left(a_{i}(z)\right) \xi_{i}(z) .
$$

Equation (2.19b) should hold for all eigenvalues $\alpha_{1}(z), \ldots, \alpha_{m}(z)$. Thus, we get

$$
\begin{gathered}
\boldsymbol{p}^{(V)}(z, 0)\left[\xi_{1}(z), \ldots, \xi_{m}(z)\right. \\
=\boldsymbol{p}_{1}^{(B)}(0)\left[V^{*}\left(\alpha_{1}(z)\right) \xi_{1}(z), \ldots, V^{*}\left(\alpha_{1}(z)\right) \xi_{m}(z)\right] .
\end{gathered}
$$

Let us define an $(m \times m)$ matrix $\Xi(z)$ of the eigenvectors as follows:

$$
\Xi(z)=\left[\xi_{1}(z), \xi_{2}(z), \ldots, \xi_{m}(z)\right] .
$$

It is proven in Lee et al. [4] that the eigenvalues $\alpha_{1}(z), \alpha_{2}(z), \ldots, \alpha_{m}(z)$ are distinct for a $z$ on $\{z:|z| \leq 1\}$, and the inverse matrix $\Xi^{-1}(z)$ exists. Thus, $(2.1 c)$ becomes

$$
\boldsymbol{p}^{(V)}(z, 0)=\boldsymbol{p}_{1}^{(B)}(0) \Xi(z) \boldsymbol{D}_{g}\left\{V^{*}\left(\alpha_{i}(z)\right)\right\}_{i=1}^{m} \Xi^{-1}(z)
$$

where

$$
\boldsymbol{D}_{g}\left\{a_{i}\right\}_{i=1}^{m}=\left(\begin{array}{cccc}
a_{1} & 0 & \ldots & 0 \\
0 & a_{2} & \ldots & 0 \\
\vdots & \vdots & \ddots & \vdots \\
0 & 0 & \ldots & a_{m}
\end{array}\right)
$$

Using $(2.19 d)$ in (2.15) yields

$$
\boldsymbol{p}^{*(V)}(z, \theta)(\theta \boldsymbol{I}+\boldsymbol{C}+z \boldsymbol{D})=\boldsymbol{p}_{1}^{(B)}(0) \Xi(z) \boldsymbol{D}_{g}\left\{V^{*}\left(\alpha_{i}(z)\right)-V^{*}(\theta)\right\}_{i=1}^{m} \Xi^{-1}(z)
$$

In the same way, we get, from (2.14) and (2.18),

$$
\begin{array}{r}
\boldsymbol{p}^{(B)}(z, 0)=\left[\boldsymbol{p}^{(S)}(z, 0)-\boldsymbol{p}_{1}^{(B)}(0)\right] \Xi(z) \boldsymbol{D}_{g}\left\{\frac{z S^{*}\left(\alpha_{i}(z)\right)}{z-S^{*}\left(\alpha_{i}(z)\right)}\right\}_{i=1}^{m} \Xi^{-1}(z) \\
\boldsymbol{p}^{(S)}(z, 0)=\left[\boldsymbol{p}^{(D)}(z)(\boldsymbol{C}+z \boldsymbol{D})+\boldsymbol{p}^{(V)}(z, 0)\right] \Xi(z) \boldsymbol{D}_{g}\left\{\left\{H^{*} a_{i}(z)\right)\right\}_{i=1}^{m} \Xi^{-1}(z) .
\end{array}
$$

Using (2.21) in (2.14) and (2.22) in (2.18), we get

$$
\begin{gathered}
\boldsymbol{p}^{*(S)}(z, \theta)(\theta \boldsymbol{I}+\boldsymbol{C}+z \boldsymbol{D})=\left[\boldsymbol{p}^{(D)}(z)(\boldsymbol{C}+z \boldsymbol{D})+\boldsymbol{p}^{(V)}(z, 0)\right] \\
\times \Xi(z) \boldsymbol{D}_{g}\left\{H^{*}\left(\alpha_{i}(z)\right)-H^{*}(\theta)\right\}_{i=1}^{m} \Xi^{-1}(z) \\
\boldsymbol{p}^{*(B)}(z, \theta)(\theta \boldsymbol{I}+\boldsymbol{C}+z \boldsymbol{D})=\left[\boldsymbol{p}^{(S)}(z, 0)-\boldsymbol{p}_{1}^{(B)}(0)\right] \\
\times \Xi(z) \boldsymbol{D}_{g}\left\{\frac{z\left[S^{*}\left(\alpha_{i}(z)\right)-S^{*}(\theta)\right]}{z-S^{*}\left(\alpha_{i}(z)\right)}\right\}_{i=1}^{m} \Xi^{-1}(z) .
\end{gathered}
$$

Equations (2.20), (2.23) and (2.24) will play central roles in obtaining the vector generating functions of the queue length and the Laplace-Stieltjes transform (LST) of the waiting time. 
All the above quantities are expressed in eigenvalues, eigenvectors and diagonal matrices of the functions of the eigenvalues. These quantities can be converted into familiar matrixanalytic notations. See Appendix I for the conversions.

\subsection{The Queue Length at an Arbitrary Time}

The vector generating function $\boldsymbol{Y}(z)$ of the queue length at an arbitrary time can be obtained from

$$
\boldsymbol{Y}(z)=\boldsymbol{p}^{(D)}(z)+\left.\boldsymbol{p}^{*(B)}(z, \theta)\right|_{\theta=0}+\left.\boldsymbol{p}^{*(V)}(z, \theta)\right|_{\theta=0}+\left.\boldsymbol{p}^{*(S)}(z, \theta)\right|_{\theta=0} .
$$

Using (2.20), (2.23) and (2.24) in (2.25), we get, after using ( $I$-2) of Appendix I,

$$
\begin{gathered}
\boldsymbol{Y}(z)=\left[\boldsymbol{p}^{(D)}(z) \Xi(z) \boldsymbol{D}_{g}\left\{H^{*}\left(\alpha_{i}(z)\right)\right\}_{i=1}^{m} \Xi^{-1}(z)\right. \\
\left.+\boldsymbol{p}_{1}^{(B)}(0) \Xi(z) \boldsymbol{D}_{g}\left\{\frac{1-V^{*}\left(\alpha_{i}(z)\right) H^{*}\left(\alpha_{i}(z)\right)}{\alpha_{i}(z)}\right\}_{i=1}^{m} \Xi^{-1}(z)\right] \\
\times \Xi(z) \boldsymbol{D}_{g}\left\{\frac{(1-z) S^{*}\left(\alpha_{i}(z)\right)}{\left.S^{*}\left(\alpha_{i}(z)\right)-z\right)}\right\}_{i=1}^{m} \Xi^{-1}(z) .
\end{gathered}
$$

The number of customers at an arbitrary time during a dormant period can be obtained from the information concerning the previous busy period termination point and the number of customers that arrive during the vacation. Thus, we can express $\boldsymbol{p}^{(D)}(z)$ in (2.26) in terms of $\boldsymbol{p}_{1}^{(B)}(0)$. Let $\left(\boldsymbol{V}_{n}\right)_{i j}$ be the probability that $n$ customers arrive during a vacation and the phase of the UMC is $j$ at the end of the vacation, given that the UMC is $i$ at the start of the vacation. Let $\boldsymbol{V}_{n}$ be the matrix of $\left(\boldsymbol{V}_{n}\right)_{i j}$ and $\boldsymbol{V}(z)$ be the matrix generating function of $\boldsymbol{V}_{n}$ such that $\boldsymbol{V}(z)=\sum_{n=0}^{\infty} \boldsymbol{V}_{n} z^{n}$. Then, we have the following theorem.

Theorem 1: If we define the matrix $\boldsymbol{\Phi}_{n}$ as

$$
\boldsymbol{\Phi}_{0}=\boldsymbol{V}_{0}, \boldsymbol{\Phi}_{n}=\sum_{k=0}^{n} \boldsymbol{V}_{k}\left[(-\boldsymbol{C})^{-1} \boldsymbol{D}\right]^{n-k}, \quad(n \geq 1),
$$

(a) we get

$$
\boldsymbol{p}^{(D)}(z)=\boldsymbol{p}_{1}^{(B)}(0) \sum_{n=0}^{N-1} \boldsymbol{\Phi}_{n}(-\boldsymbol{C})^{-1} z^{n}
$$

(b) $\quad\left(\mathbf{\Phi}_{n}\right)_{i j}$ is the probability that the system state (queue length) visits $n$ during the dormant period and the phase of the UMC at the visit is $j$, given that the vacation starts with the phase in $i$.

Proof: (a) For $n=0$, we get, from (2.1), $-\boldsymbol{p}_{0}^{(D)} \boldsymbol{C}=\boldsymbol{p}_{0}^{(V)}(0)$. The queue length at the end of the vacation is the number of customers that arrive during the vacation. Thus, we get $\boldsymbol{p}^{(V)}(z, 0)=\boldsymbol{p}_{1}^{(B)}(0) \boldsymbol{V}(z)$ which means $\boldsymbol{p}_{n}^{(V)}(0)=\boldsymbol{p}_{1}^{(B)}(0) \boldsymbol{V}_{n}$. With $n=0$, we get $\boldsymbol{p}_{0}^{(V)}(0)=\boldsymbol{p}_{1}^{(B)}(0) \boldsymbol{V}_{0}$, which implies $-\boldsymbol{p}_{0}^{(D)} \boldsymbol{C}=\boldsymbol{p}_{1}^{(B)}(0) \boldsymbol{V}_{0}=\boldsymbol{p}_{1}^{(B)}(0) \boldsymbol{\Phi}_{0}$ from the definition. Thus we get $\boldsymbol{p}_{0}^{(D)}=\boldsymbol{p}_{1}^{(B)}(0) \boldsymbol{\Phi}_{0}(-\boldsymbol{C})^{-1}$. For $n \geq 1$, we get, from (2.2),

$$
-\boldsymbol{p}_{n}^{(D)} \boldsymbol{C}=\boldsymbol{p}_{1}^{(B)}(0) \sum_{k=0}^{n} \boldsymbol{V}_{k}\left[(-\boldsymbol{C})^{-1} \boldsymbol{D}\right]^{n-k}=\boldsymbol{p}_{1}^{(B)}(0) \boldsymbol{\Phi}_{n} .
$$

Thus, we get $\boldsymbol{p}_{n}^{(D)}=\boldsymbol{p}_{1}^{(B)}(0) \boldsymbol{\Phi}_{n}(-\boldsymbol{C})^{-1}$, which implies (2.28). 
(b) We first notice that $(-\boldsymbol{C})^{-1} \boldsymbol{D}$ is the phase change probability during an interarrival time. Conditioning on the number of customers that arrive during the vacation completes the proof.

Using (2.28) in (2.26), $Y(z)$ becomes

$$
\begin{gathered}
\boldsymbol{Y}(z)=\boldsymbol{p}_{1}^{(B)}(0)\left[\sum_{n=0}^{N-1} \boldsymbol{\Phi}_{n}(-\boldsymbol{C})^{-1} z^{n} \Xi(z) \boldsymbol{D}_{g}\left\{H^{*}\left(\alpha_{i}(z)\right)\right\}_{i=1}^{m} \Xi^{-1}(z)\right. \\
\left.+\Xi(z) \boldsymbol{D}_{g}\left\{\frac{1-V^{*}\left(\alpha_{i}(z)\right) H^{*}\left(\alpha_{i}(z)\right)}{\alpha_{i}(z)}\right\}_{i=1}^{m} \Xi^{-1}(z)\right] \\
\times \Xi(z) \boldsymbol{D}_{g}\left\{\frac{(1-z) S^{*}\left(\alpha_{i}(z)\right)}{\left.S^{*}\left(\alpha_{i}(z)\right)-z\right)}\right\}_{i=1}^{m} \Xi^{-1}(z) .
\end{gathered}
$$

Now, we consider the term $\boldsymbol{p}_{1}^{(B)}(0)$ contained in (2.29). We note that $\boldsymbol{p}_{1}^{(B)}(0)$ is the rate at which the system becomes empty. If we let $\boldsymbol{x}_{0}$ be the vector probability that there are no customers in the system at an arbitrary departure, this should be equal to $\lambda \boldsymbol{x}_{0}$ because $\lambda$, which is the arrival rate, should be equal to the departure rate in steady state. Thus, we have

$$
\boldsymbol{p}_{1}^{(B)}(0)=\lambda \boldsymbol{x}_{0}
$$

Using (2.30) in (2.29), we get

$$
\begin{aligned}
& \boldsymbol{Y}(z)=\lambda \boldsymbol{x}_{0}\left[\sum_{n=0}^{N-1} \boldsymbol{\Phi}_{n}(-\boldsymbol{C})^{-1} z^{n} \Xi(z) \boldsymbol{D}_{g}\left\{H^{*}\left(\alpha_{i}(z)\right)\right\}_{i=1}^{m} \Xi^{-1}(z)\right. \\
&+\left.\Xi(z) \boldsymbol{D}_{g}\left\{\frac{1-V^{*}\left(\alpha_{i}(z)\right) H^{*}\left(\alpha_{i}(z)\right)}{\alpha_{i}(z)}\right\}_{i=1}^{m} \Xi^{-1}(z)\right] \\
& \times \Xi(z) \boldsymbol{D}_{g}\left\{\frac{(1-z) S^{*}\left(\alpha_{i}(z)\right)}{\left.S^{*}\left(\alpha_{i}(z)\right)-z\right)}\right\}_{i=1}^{m} \Xi^{-1}(z) .
\end{aligned}
$$

Now, using Appendix I, we can convert (2.31a) into the matrix-analytic notations as follows:

$$
\begin{gathered}
\boldsymbol{Y}(z)=\lambda(z-1) \boldsymbol{x}_{0}\left\{\sum_{n=0}^{N-1} \boldsymbol{\Phi}_{n}(-\boldsymbol{C})^{-1} z^{n} \boldsymbol{H}(z)+[\boldsymbol{V}(z) \boldsymbol{H}(z)-\boldsymbol{I}](\boldsymbol{C}+z \boldsymbol{D})^{-1}\right\} \\
\times \boldsymbol{A}(z)[z \boldsymbol{I}-\boldsymbol{A}(z)]^{-1}
\end{gathered}
$$

where $\boldsymbol{A}(z)$ and $\boldsymbol{H}(z)$ are the matrix generating functions of the number of customers that arrive during a service time and a set-up time, respectively.

Now, we need to find $\boldsymbol{x}_{0}$. Obtaining $\boldsymbol{x}_{0}$ requires the distribution of the number of customers that are served between two neighboring busy-period termination points. (We will call this interval a cycle.) This, in turn, requires the queue length distribution at the busy period initiation point. 
Theorem 2: Let $\boldsymbol{Q}_{N, \text { setup }}(z)$ be the matrix generating function of the queue length at the starting epoch of an arbitrary busy period, given the phase at the ending epoch of the previous busy period. Then, we have

$$
\boldsymbol{Q}_{N, \text { setup }}(z)=\sum_{n=0}^{N-1} \boldsymbol{\Phi}_{n}(-\boldsymbol{C})^{-1} z^{n}(\boldsymbol{C}+z \boldsymbol{D}) \boldsymbol{H}(z)+\boldsymbol{V}(z) \boldsymbol{H}(z) .
$$

Proof: Let $\boldsymbol{Q}_{N}(z)$ be the matrix generating function of the queue length at an arbitrary busy period starting epoch of the MAP/G/1 queue with $N$-policy and a single vacation (without set-up). Conditioning on the number of customers that arrive during the vacation, we get

$$
\boldsymbol{Q}_{N}(z)=\boldsymbol{V}(z)-\sum_{n=0}^{N-1} \boldsymbol{V}_{n} z^{n}+\sum_{k=0}^{N-1} \boldsymbol{V}_{k}\left[(-\boldsymbol{C})^{-1} \boldsymbol{D}\right]^{N-k} z^{N} .
$$

From Theorem 1, we have

$$
\begin{aligned}
& \sum_{n=0}^{N-1} \boldsymbol{\Phi}_{n}(-\boldsymbol{C})^{-1} z^{n}=\sum_{n=0}^{N-1} \sum_{k=0}^{n} \boldsymbol{V}_{k}\left[(-\boldsymbol{C})^{-1} \boldsymbol{D}\right]^{n-k}(-\boldsymbol{C})^{-1} z^{n} \\
= & \sum_{n=0}^{N-1} \boldsymbol{V}_{n}(-\boldsymbol{C})^{-1} z^{n}+\sum_{n=1}^{N-1} \sum_{k=0}^{n-1} \boldsymbol{V}_{k}\left[(-\boldsymbol{C})^{-1} \boldsymbol{D}\right]^{n-k}(-\boldsymbol{C})^{-1} z^{n} .
\end{aligned}
$$

Postmultiplying both sides of the above equation by $(-C)$, we get

$$
\sum_{n=0}^{N-1} \boldsymbol{V}_{n} z^{n}=\sum_{n=0}^{N-1} \boldsymbol{\Phi}_{n} z^{n}-\sum_{n=1}^{N-1} \sum_{k=0}^{n-1} \boldsymbol{V}_{k}\left[(-\boldsymbol{C})^{-1} \boldsymbol{D}\right]^{n-k} z^{n} .
$$

Using (2.34) in (2.33), we have

$$
\begin{gathered}
\boldsymbol{Q}_{N}(z)=\boldsymbol{V}(z)-\left\{\sum_{n=0}^{N-1} \boldsymbol{\Phi}_{n} z^{n}-\sum_{n=1}^{N-1} \sum_{k=0}^{n-1} \boldsymbol{V}_{k}\left[(-\boldsymbol{C})^{-1} \boldsymbol{D}\right]^{n-k} z^{n}\right\} \\
+\sum_{k=0}^{N-1} \boldsymbol{V}_{k}\left[(-\boldsymbol{C})^{-1} \boldsymbol{D}\right]^{N-k} z^{N} \\
=\boldsymbol{V}(z)-\sum_{n=0}^{N-1} \boldsymbol{\Phi}_{n} z^{n}+\sum_{n=1}^{N} \sum_{k=0}^{n-1} \boldsymbol{V}_{k}\left[(-\boldsymbol{C})^{-1} \boldsymbol{D}\right]^{n-k} z^{n} \\
=\boldsymbol{V}(z)+\sum_{n=0}^{N-1} \boldsymbol{\Phi}_{n}(-\boldsymbol{C})^{-1} z^{n} \boldsymbol{C}+\left\{\sum_{m=0}^{N-1} \sum_{k=0}^{m} \boldsymbol{V}_{k}\left[(-\boldsymbol{C})^{-1} \boldsymbol{D}\right]^{m-k}(-\boldsymbol{C})^{-1} z^{m}\right\} \boldsymbol{D} z .
\end{gathered}
$$

Using (2.27), we get

$$
\boldsymbol{Q}_{N}(z)=\sum_{n=0}^{N-1} \boldsymbol{\Phi}_{n}(-\boldsymbol{C})^{-1} z^{n}(\boldsymbol{C}+\boldsymbol{D} z)+\boldsymbol{V}(z)
$$


Then, $\boldsymbol{Q}_{N \text {,setup }}(z)=\boldsymbol{Q}_{N}(z) \boldsymbol{H}(z)$ completes the proof.

Now, define $\boldsymbol{K}(z)$ as the matrix generating function of the number of customers that are served during a cycle. Then using (2.32), we have

$$
\begin{gathered}
\boldsymbol{K}(z)=\left.\boldsymbol{Q}_{N, \text { setup }}(z)\right|_{z=\boldsymbol{G}(z)} \\
=\left[\sum_{n=0}^{N-1} \boldsymbol{\Phi}_{n}(-\boldsymbol{C})^{-1}[\boldsymbol{G}(z)]^{n}[\boldsymbol{C}+\boldsymbol{D} \boldsymbol{G}(z)]+\boldsymbol{V}(\boldsymbol{G}(z))\right] \boldsymbol{H}(\boldsymbol{G}(z))
\end{gathered}
$$

where $\boldsymbol{V}(\boldsymbol{G}(z))=\int_{0}^{\infty} e^{(C+D G(z)) x} d V(x)$ (Lucantoni, et al. [10]) and $\boldsymbol{G}(z)$ is the matrix generating function of the number of customers that are served during a fundamental period (Neuts [12]).

Let $\boldsymbol{K}=\left.\boldsymbol{K}(z)\right|_{z=1}$ be the matrix of the phase change probability of the UMC during an arbitrary cycle. Then, from (2.36) we get

$$
\boldsymbol{K}=\left.\boldsymbol{K}(z)\right|_{z=1}=\left[\sum_{n=0}^{N-1} \boldsymbol{\Phi}_{n}(-\boldsymbol{C})^{-1} \boldsymbol{G}^{n}(\boldsymbol{C}+\boldsymbol{D} \boldsymbol{G})+\boldsymbol{V}(\boldsymbol{G})\right] \boldsymbol{H}(\boldsymbol{G}) .
$$

Let $\kappa$ be the stationary vector of $K$ such that $\kappa=\kappa K$ and $\kappa \boldsymbol{e}=1$. Let $\boldsymbol{\kappa}^{*}=\left.\frac{d}{d z} \boldsymbol{K}(z)\right|_{z=1} \boldsymbol{e}$ be the mean number of customers that are served during a cycle, given the phase at the ending epoch of a busy period. Then, it is well known that $\boldsymbol{x}_{0}$ can be obtained from (Neuts [12])

$$
x_{0}=\frac{\kappa}{\kappa \kappa^{*}} .
$$

Now, we can express $\boldsymbol{x}_{0}$ in terms of $\boldsymbol{\Phi}_{n}$ defined and interpreted in Theorem 1. We first obtain $\kappa^{*}$.

Theorem 3: We have

$$
\begin{gathered}
\boldsymbol{\kappa}^{*}=\frac{\lambda}{1-\rho}\left[\sum_{n=0}^{N-1} \boldsymbol{\Phi}_{n}(-\boldsymbol{C})^{-1}+E(V) \boldsymbol{I}+E(H) \boldsymbol{I}\right] \boldsymbol{e} \\
+(\boldsymbol{K}-\boldsymbol{I})(\boldsymbol{e} \boldsymbol{g}+\boldsymbol{C}+\boldsymbol{D} \boldsymbol{G})^{-1} \boldsymbol{D} \boldsymbol{\mu}
\end{gathered}
$$

Proof: See Appendix III.

Using (2.39) in (2.38) with $\boldsymbol{\kappa}(\boldsymbol{K}-\boldsymbol{I})=\mathbf{0}$ yields

$$
\boldsymbol{x}_{0}=\frac{(1-\rho) \boldsymbol{\kappa}}{\lambda\left[\sum_{n=0}^{N-1} \boldsymbol{\Phi}_{n}(-C)^{-1} \boldsymbol{e}+E(V)+E(H)\right]} .
$$

Using (2.40) in $(2.31 a, b)$, we get

$$
\begin{aligned}
\boldsymbol{Y}(z) & =\boldsymbol{r}_{1}(z) \cdot \Xi(z) \boldsymbol{D}_{g}\left\{\frac{(1-\rho)(1-z) S^{*}\left(\alpha_{i}(z)\right)}{S^{*}\left(\alpha_{i}(z)\right)-z}\right\}_{i=1}^{m} \Xi^{-1}(z) \\
& =\boldsymbol{r}_{2}(z)(1-\rho)(z-1) \boldsymbol{A}(z)[z \boldsymbol{I}-\boldsymbol{A}(z)]^{-1}
\end{aligned}
$$


where

$$
\begin{gathered}
\boldsymbol{r}_{1}(z)=\frac{1}{\boldsymbol{\kappa} \sum_{n=0}^{N-1} \boldsymbol{\Phi}_{n}(-\boldsymbol{C})^{-1} \boldsymbol{e}+E(V)+E(H)} \\
\times\left[\boldsymbol{\kappa} \sum_{n=0}^{N-1} \boldsymbol{\Phi}_{n}(-\boldsymbol{C})^{-1} z^{n}+E(V) \boldsymbol{\kappa} \Xi(z) \boldsymbol{D}_{g}\left\{\frac{1-V^{*}\left(\alpha_{i}(z)\right)}{E(V) \alpha_{i}(z)}\right\}_{i=1}^{m} \Xi^{-1}(z)\right. \\
\left.+E(H) \boldsymbol{\kappa}\left\{\sum_{n=0}^{N-1} \boldsymbol{\Phi}_{n} z^{n}(\boldsymbol{C}+\boldsymbol{D} z)+\boldsymbol{V}(z)\right\} \Xi(z) \boldsymbol{D}_{g}\left\{\frac{1-H^{*}\left(\alpha_{i}(z)\right)}{E(H) \alpha_{i}(z)}\right\}_{i=1}^{m} \Xi^{-1}(z)\right]
\end{gathered}
$$

and

$$
\begin{gathered}
\boldsymbol{r}_{2}(z)=\frac{1}{\boldsymbol{\kappa} \sum_{n=0}^{N-1} \boldsymbol{\Phi}_{n}(-\boldsymbol{C})^{-1} \boldsymbol{e}+E(V)+E(H)} \\
\times\left[\boldsymbol{\kappa} \sum_{n=0}^{N-1} \boldsymbol{\Phi}_{n}(-\boldsymbol{C})^{-1} z^{n}+E(V) \boldsymbol{\kappa} \frac{[\boldsymbol{V}(z)-\boldsymbol{I}](\boldsymbol{C}+z \boldsymbol{D})^{-1}}{E(V)}\right. \\
\left.+E(H) \boldsymbol{\kappa}\left\{\sum_{n=0}^{N-1} \boldsymbol{\Phi}_{n} z^{n}(\boldsymbol{C}+\boldsymbol{D} z)+\boldsymbol{V}(z)\right\} \frac{[\boldsymbol{H}(z)-\boldsymbol{I}](\boldsymbol{C}+z \boldsymbol{D})^{-1}}{E(H)}\right] .
\end{gathered}
$$

We note that $\boldsymbol{r}_{2}(z)$ is just the matrix-analytic expression of $\boldsymbol{r}_{1}(z)$ and they are equivalent.

\subsection{The Queue Length at Departures}

All the information concerning the queue length at departures can be recover from $\boldsymbol{p}^{(B)}(z, 0)$, because the departure epochs are the epochs at which the remaining service times become zero. Thus, after a normalization and discounting the departing customer, we get the vector generating function $\boldsymbol{X}(z)$ of the queue length at departures as

$$
\boldsymbol{X}(z)=\frac{\boldsymbol{p}^{(B)}(z, 0)}{\boldsymbol{p}^{(B)}(1,0) \boldsymbol{e}} z^{-1}=\frac{\boldsymbol{p}^{(B)}(z, 0)}{\lambda} z^{-1}
$$

where we used $\lambda=\boldsymbol{p}^{(B)}(1,0) \boldsymbol{e}=\left.\boldsymbol{p}^{(B)}(z, 0)\right|_{z=1} \boldsymbol{e}$, because $\boldsymbol{p}^{(B)}(1,0) \boldsymbol{e}=\sum_{n=1}^{\infty} \boldsymbol{p}_{n}^{(B)}(0) \boldsymbol{e}$ is the departure rate of the customers which is equal to the arrival rate in steady state.

After using (2.19d), (2.22), (2.28) and (2.30) in (2.21), we can rewrite $\boldsymbol{p}^{(B)}(z, 0)$ in a different form as

$$
\begin{aligned}
\boldsymbol{p}^{(B)}(z, 0)= & \lambda \boldsymbol{x}_{0}\left[\sum_{n=0}^{N-1} \boldsymbol{\Phi}_{n}(-\boldsymbol{C})^{-1} z^{n} \Xi(z) \boldsymbol{D}_{g}\left\{H^{*}\left(\alpha_{i}(z)\right)\right\}_{i=1}^{m} \Xi^{-1}(z)\right. \\
+ & \left.\Xi(z) \boldsymbol{D}_{g}\left\{\frac{1-V^{*}\left(\alpha_{i}(z)\right) H^{*}\left(\alpha_{i}(z)\right)}{\alpha_{i}(z)}\right\}_{i=1}^{m} \Xi^{-1}(z)\right] \\
& \times \Xi(z) \boldsymbol{D}_{g}\left\{\frac{z \alpha_{i}(z) S^{*}\left(\alpha_{i}(z)\right)}{S^{*}\left(\alpha_{i}(z)\right)-z}\right\}_{i=1}^{m} \Xi^{-1}(z) .
\end{aligned}
$$

Using (2.45) in (2.44) together with (2.40), we get 


$$
\begin{gathered}
\boldsymbol{X}(z)=\boldsymbol{r}_{1}(z) \cdot \Xi(z) \boldsymbol{D}_{g}\left\{\frac{(1-\rho) \alpha_{i}(z) S^{*}\left(\alpha_{i}(z)\right)}{\lambda\left[S^{*}\left(\alpha_{i}(z)\right)-z\right]}\right\}_{i=1}^{m} \Xi^{-1}(z) \\
=\boldsymbol{r}_{2}(z) \frac{(1-\rho)}{\lambda}(\boldsymbol{C}+z \boldsymbol{D}) \boldsymbol{A}(z)[z \boldsymbol{I}-\boldsymbol{A}(z)]^{-1}
\end{gathered}
$$

where $\boldsymbol{r}_{1}(z)$ and $\boldsymbol{r}_{2}(z)$ were given in (2.42) and (2.43).

From (2.41) and (2.46), we confirm the well known relationship (Takine and Takahashi [18]),

$$
\boldsymbol{Y}(z)(\boldsymbol{C}+z \boldsymbol{D})=\lambda(z-1) \boldsymbol{X}(z) .
$$

\subsection{Decompositions and Probabilistic Interpretations}

To interpret $\boldsymbol{r}_{1}(z)$ (or equivalently, $\boldsymbol{r}_{2}(z)$ ), we need the mean length of the idle period $I_{N \text {,setup }}$ which consists of a vacation, a dormant period and a set-up time. Let $\boldsymbol{T}_{n}^{*}(\theta)$ be the matrix LST of the time until $n$ customers arrive given the phase of the UMC at time 0 . Then, we easily get

$$
\boldsymbol{T}_{n}^{*}(\theta)=(\theta \boldsymbol{I}-\boldsymbol{C})^{-1} \boldsymbol{D} \boldsymbol{T}_{n-1}^{*}(\theta),(n \geq 1),\left(\boldsymbol{T}_{0}^{*}(\theta)=\boldsymbol{I}\right)
$$

where $(\theta \boldsymbol{I}-\boldsymbol{C})^{-1} \boldsymbol{D}$ is the LST of the time length until the first arrival. Let $\boldsymbol{I}_{N}^{*}(\theta)$ be the matrix Laplace transform of the idle period of the MAP/G/1 queue under $N$-policy with a single vacation (without set-up), given the phase at the end of a busy period. Then, conditioning on the number of arrivals during the vacation, we get

$$
\boldsymbol{I}_{N}^{*}(\theta)=\sum_{k=0}^{N-1} \boldsymbol{V}_{k}^{*}(\theta) \boldsymbol{T}_{N-k}^{*}(\theta)+\sum_{k=N}^{\infty} \boldsymbol{V}_{k}^{*}(\theta)
$$

where $V_{k}^{*}(\theta)$ is the matrix Laplace transform of the vacation length which includes the probability that $k$ customers arrive during the vacation.

Let $\tau_{n, i}$ be the mean time until $n$ customers arrive, given that the UMC is in phase $i$ at time 0 . Define a column vector $\boldsymbol{\tau}_{n}=\left(\tau_{n, 1}, \tau_{n, 2}, \ldots, \tau_{n, m}\right)^{T}$. Then using (2.48), we can easily derive

$$
\boldsymbol{\tau}_{n}=-\left.\boldsymbol{T}_{n}^{*(1)}(\theta)\right|_{\theta=0} \boldsymbol{e}=\sum_{k=0}^{n-1}\left[(-\boldsymbol{C})^{-1} \boldsymbol{D}\right]^{k}(-\boldsymbol{C})^{-1} \boldsymbol{e} .
$$

Let $\eta_{N, i}$ be the mean length of the idle period in the MAP/G/1 queue under $N$-policy and a single vacation (without set-up), given that the UMC is in phase $i$ at the end of a busy period. Let us define the column vector $\boldsymbol{\eta}_{N}=\left(\eta_{N, 1}, t_{N, 2}, \ldots, \eta_{N, m}\right)^{T}$. Then, we can get

$$
\boldsymbol{\eta}_{N}=-\left.\frac{d}{d \theta} \boldsymbol{I}_{N}^{*}(\theta)\right|_{\theta=0} \boldsymbol{e}=\sum_{n=0}^{N-1} \boldsymbol{\Phi}_{n}(-\boldsymbol{C})^{-1} \boldsymbol{e}+E(V) \boldsymbol{e} .
$$

Then, from (2.49), we get

$$
E\left(I_{N}\right)=-\left.\frac{d}{d \theta} \boldsymbol{I}_{N}^{*}(\theta) \boldsymbol{e}\right|_{\theta=0}=\left[\boldsymbol{\kappa} \sum_{k=0}^{N-1} \boldsymbol{\Phi}_{k}(-\boldsymbol{C})^{-1} \boldsymbol{e}+E(V)\right] .
$$

Then, we get the mean length of an idle period as 


$$
E\left(I_{N, \text { setup }}\right)=E\left(I_{N}\right)+E(H)=\kappa \sum_{n=0}^{N-1} \boldsymbol{\Phi}_{n}(-\boldsymbol{C})^{-1} \boldsymbol{e}+E(V)+E(H)
$$

If we let $E\left(B_{N, \text { setup }}\right)$ be the mean length of an arbitrary busy period, we easily get, from (2.38) and (2.40),

$$
E\left(B_{N, \text { setup }}\right)=\boldsymbol{\kappa} \boldsymbol{\kappa}^{*} E(S)=\frac{\rho}{1-\rho}\left[\boldsymbol{\kappa} \sum_{k=0}^{N-1} \boldsymbol{\Phi}_{k}(-\boldsymbol{C})^{-1} \boldsymbol{e}+E(V)+E(H)\right]
$$

Now, we are ready to interpret the queue length generating functions given in (2.41) and (2.46). If the system state during the dormant period visits $n$, it stays there for $(-\boldsymbol{C})^{-1} \boldsymbol{e}$ on the average. Thus $\boldsymbol{\kappa} \sum_{n=0}^{N-1} \boldsymbol{\Phi}_{n}(-\boldsymbol{C})^{-1} \boldsymbol{e}$ is the mean length of the dormant period during an arbitrary idle period. Thus, without proofs, we have Theorem 4 and Theorem 5.

Theorem 4: Let $\phi^{(D)}$ and $\phi^{(V)}$ be the probabilities that the server is in a dormant period and in a set-up period given that the system is idle. Then we get

$$
\begin{aligned}
\phi^{(D)} & =\frac{\boldsymbol{\kappa} \sum_{n=0}^{N-1} \boldsymbol{\Phi}_{n}(-\boldsymbol{C})^{-1} \boldsymbol{e}}{\boldsymbol{\kappa} \sum_{n=0}^{N-1} \boldsymbol{\Phi}_{n}(-\boldsymbol{C})^{-1} \boldsymbol{e}+E(V)+E(H)} \\
\phi^{(V)} & =\frac{E(V)}{\boldsymbol{\kappa} \sum_{n=0}^{N-1} \boldsymbol{\Phi}_{n}(-\boldsymbol{C})^{-1} \boldsymbol{e}+E(V)+E(H)} \\
\phi^{(S)} & =\frac{E(H)}{\boldsymbol{\kappa} \sum_{n=0}^{N-1} \boldsymbol{\Phi}_{n}(-\boldsymbol{C})^{-1} \boldsymbol{e}+E(V)+E(H)} .
\end{aligned}
$$

Theorem 5: Let $\psi_{n, i}$ be the probability that at an arbitrary time during a dormant period, there are $n$ customers in the system and the phase of the UMC is in $i$. Define the vector $\boldsymbol{\psi}_{n}=\left(\psi_{n, 1}, \psi_{n, 2}, \ldots, \psi_{n, m}\right)$. Then we get

$$
\boldsymbol{\psi}_{n}=\frac{\boldsymbol{\kappa} \boldsymbol{\Phi}_{n}(-\boldsymbol{C})^{-1}}{\kappa \sum_{k=0}^{N-1} \boldsymbol{\Phi}_{k}(-\boldsymbol{C})^{-1} \boldsymbol{e}},(0 \leq n \leq N-1)
$$

Now, using $(2.55 a, b, c)$ and (2.56), the vector generating function $\boldsymbol{Y}(z)$ of the queue length at an arbitrary time $((2.41))$ and $\boldsymbol{X}(z)$ at departures $((2.46))$ become

$$
\begin{gathered}
\boldsymbol{Y}(z)=\left\{\phi^{(D)} \sum_{n=0}^{N-1} \boldsymbol{\psi}_{n} z^{n}+\phi^{(V)} \boldsymbol{\kappa} \boldsymbol{V}^{+}(z)+\phi^{(S)} \boldsymbol{\kappa}\left[\sum_{n=0}^{N-1} \boldsymbol{\Phi}_{n} z^{n}(\boldsymbol{C}+z \boldsymbol{D})+\boldsymbol{V}(z)\right] \boldsymbol{H}^{+}(z)\right\} \\
\times \Xi(z) \boldsymbol{D}_{g}\left\{\frac{(1-\rho)(1-z) S^{*}\left(\alpha_{i}(z)\right)}{S^{*}\left(\alpha_{i}(z)\right)-z}\right\}_{i=1}^{m} \Xi^{-1}(z) \\
=\left\{\phi^{(D)} \sum_{n=0}^{N-1} \boldsymbol{\psi}_{n} z^{n}+\phi^{(V)} \boldsymbol{\kappa} \boldsymbol{V}^{+}(z)+\phi^{(S)} \boldsymbol{\kappa}\left[\sum_{n=0}^{N-1} \boldsymbol{\Phi}_{n} z^{n}(\boldsymbol{C}+z \boldsymbol{D})+\boldsymbol{V}(z)\right] \boldsymbol{H}^{+}(z)\right\} \\
\times(1-\rho)(z-1) \boldsymbol{A}(z)[z \boldsymbol{I}-\boldsymbol{A}(z)]^{-1}
\end{gathered}
$$




$$
\begin{gathered}
\boldsymbol{X}(z)=\left\{\phi^{(D)} \sum_{n=0}^{N-1} \boldsymbol{\psi}_{n} z^{n}+\phi^{(V)} \boldsymbol{\kappa} \boldsymbol{V}^{+}(z)+\phi^{(S)} \boldsymbol{\kappa}\left[\sum_{n=0}^{N-1} \boldsymbol{\Phi}_{n} z^{n}(\boldsymbol{C}+z \boldsymbol{D})+\boldsymbol{V}(z)\right] \boldsymbol{H}^{+}(z)\right\} \\
\times \Xi(z) \boldsymbol{D}_{g}\left\{\frac{(1-\rho) \alpha_{i}(z) S^{*}\left(\alpha_{i}(z)\right)}{\lambda\left[S^{*}\left(\alpha_{i}(z)\right)-z\right]}\right\}_{i=1}^{m} \Xi^{-1}(z) \\
=\left\{\phi^{(D)} \sum_{n=0}^{N-1} \boldsymbol{\psi}_{n} z^{n}+\phi^{(V)} \boldsymbol{\kappa} \boldsymbol{V}^{+}(z)+\phi^{(S)} \boldsymbol{\kappa}\left[\sum_{n=0}^{N-1} \boldsymbol{\Phi}_{n} z^{n}(\boldsymbol{C}+z \boldsymbol{D})+\boldsymbol{V}(z)\right] \boldsymbol{H}^{+}(z)\right\} \\
\times \frac{(1-\rho)}{\lambda}(\boldsymbol{C}+z \boldsymbol{D}) \boldsymbol{A}(z)[z \boldsymbol{I}-\boldsymbol{A}(z)]^{-1}
\end{gathered}
$$

where $\boldsymbol{V}^{+}(z)$ and $\boldsymbol{H}^{+}(z)$ are the matrix generating functions of the number of customers that arrive during a vacation time and a set-up time, respectively (see Appendix I).

Now, we are ready to interpret the term

$$
\left\{\phi^{(D)} \sum_{n=0}^{N-1} \boldsymbol{\psi}_{n} z^{n}+\phi^{(V)} \boldsymbol{\kappa} \boldsymbol{V}^{+}(z)+\phi^{(S)} \boldsymbol{\kappa}\left[\sum_{n=0}^{N-1} \boldsymbol{\Phi}_{n} z^{n}(\boldsymbol{C}+z \boldsymbol{D})+\boldsymbol{V}(z)\right] \boldsymbol{H}^{+}(z)\right\}
$$

which is common to (2.57) and (2.58). In the following theorem, we show that (2.59) is the vector generating function of the queue length at an arbitrary idle time.

Theorem 6: Let $\boldsymbol{p}_{\text {idle }}(z)$ be the vector generating function of the queue length at an arbitrary idle time. Then we have

$\boldsymbol{p}_{\text {idle }}(z)=$

$$
\left\{\phi^{(D)} \sum_{n=0}^{N-1} \boldsymbol{\psi}_{n} z^{n}+\phi^{(V)} \boldsymbol{\kappa} \boldsymbol{V}^{+}(z)+\phi^{(S)} \boldsymbol{\kappa}\left[\sum_{n=0}^{N-1} \boldsymbol{\Phi}_{n} z^{n}(\boldsymbol{C}+z \boldsymbol{D})+\boldsymbol{V}(z)\right] \boldsymbol{H}^{+}(z)\right\}
$$

Proof: From Theorem 4, the system is in a dormant period with probability $\phi^{(D)}$, in a vacation with probability $\phi^{(V)}$, or in a set-up period with probability $\phi^{(S)}$. If the system is in a dormant period, there are $n$ customers in the system with probability $\boldsymbol{\psi}_{n}$ which accounts for the first term. If the system is in a vacation at an arbitrary time, the queue length is the number of customers that arrive during the elapsed vacation time, which accounts for the second term. If the system is in a set-up period, the queue length is the number of customers at the set-up period starting point (see Equation (2.35) for this), plus the number of customers that arrive during the elapsed set-up time, which accounts for the third term.

Applying (2.60) into (2.57) and (2.58), we get the final decomposed forms of the vector generating function of the queue length distributions as

$$
\begin{aligned}
& \boldsymbol{Y}(z)=\boldsymbol{p}_{\text {idle }}(z) \cdot \chi_{Y}(z) \\
& \boldsymbol{X}(z)=\boldsymbol{p}_{\text {idle }}(z) \cdot \chi_{X}(z)
\end{aligned}
$$

where 


$$
\begin{gathered}
\chi_{Y}(z)=\Xi(z) \boldsymbol{D}_{g}\left\{\frac{(1-\rho)(1-z) S^{*}\left(\alpha_{i}(z)\right)}{S^{*}\left(\alpha_{i}(z)\right)-z}\right\}_{i=1}^{m} \Xi^{-1}(z) \\
=(1-\rho)(z-1) \boldsymbol{A}(z)[z \boldsymbol{I}-\boldsymbol{A}(z)]^{-1}
\end{gathered}
$$

and

$$
\begin{gathered}
\boldsymbol{\chi}_{X}(z)=\Xi(z) \boldsymbol{D}_{g}\left\{\frac{(1-\rho) \alpha_{i}(z) S^{*}\left(\alpha_{i}(z)\right)}{\lambda\left[S^{*}\left(\alpha_{i}(z)\right)-z\right]}\right\}_{i=1}^{m} \Xi^{-1}(z) \\
=\frac{(1-\rho)}{\lambda}(\boldsymbol{C}+z \boldsymbol{D}) \boldsymbol{A}(z)[z \boldsymbol{I}-\boldsymbol{A}(z)]^{-1} .
\end{gathered}
$$

Decomposition results of (2.61) and (2.62) confirm the results of Lee and Ahn [5, 6].

\section{The Waiting Time}

In this section, we derive the LST of the waiting time of an arbitrary arriving customer (actual waiting time). Let us define the following notations.

$W_{A}^{*}(\theta)$ : the LST of the actual waiting time of an arbitrary arriving customer

$W_{A}^{*(B)}(\theta)$ : the LST of the actual waiting time of the customer who arrives during the busy period (including the probability that the server is busy when the customer arrives)

$W_{A}^{*(D)}(\theta): \quad$ the LST of the actual waiting time of the customer who arrives during the dormant period (including the probability that the server is in a dormant period when the customer arrives)

$W_{A}^{*(V)}(\theta)$ : the LST of the actual waiting time of the customer who arrives during the vacation (including the probability that the server is busy when the customer arrives).

First, we obtain $W_{A}^{*(B)}(\theta)$. The virtual waiting time in this case is the sum of

(i) the remaining service and

(ii) the service times of the waiting customers.

Information concerning $(i)$ and $(i i)$ is contained in $\boldsymbol{p}^{*(B)}(z, \theta)$ in (2.24). Using

(2.22), (2.30), (2.40) and $(\theta \boldsymbol{I}+\boldsymbol{C}+z \boldsymbol{D})^{-1}=\Xi(z) \boldsymbol{D}_{g}\left\{\frac{1}{\theta-\alpha_{i}(z)}\right\}_{i=1}^{m} \Xi^{-1}(z)$, we can rewrite (2.24) as

$\boldsymbol{p}^{*(B)}(z, \theta)=(1-\rho)$

$$
\begin{gathered}
\times\left[\phi^{(C)} \sum_{n=0}^{N-1} \boldsymbol{\psi}_{n} z^{n} \Xi(z) \boldsymbol{D}_{g}\left\{\frac{\alpha_{i}(z) H^{*}\left(\alpha_{i}(z)\right)}{\theta-\alpha_{i}(z)}\right\}_{i=1}^{m} \Xi^{-1}(z)\right. \\
\left.+\phi^{(V)} \boldsymbol{\kappa} \Xi(z) \boldsymbol{D}_{g}\left\{\frac{1-V^{*}\left(\alpha_{i}(z)\right) H^{*}\left(\alpha_{i}(z)\right)}{E(V)\left[\theta-\alpha_{i}(z)\right]}\right\}_{i=1}^{m} \Xi^{-1}(z)\right] \\
\times \Xi(z) \boldsymbol{D}_{g}\left\{\frac{z\left[S^{*}\left(\alpha_{i}(z)\right)-S^{*}(\theta)\right]}{S^{*}\left(\alpha_{i}(z)\right)-z}\right\}_{i=1}^{m} \Xi^{-1}(z) .
\end{gathered}
$$

Thus, the LST $W_{A}^{*(B)}(\theta)$ becomes 


$$
\begin{gathered}
W_{A}^{*(B)}(\theta)=\left.\frac{\boldsymbol{p}^{*(B)}(z, \theta)}{z}\right|_{z=S^{*}(\theta)} \frac{\boldsymbol{D}}{\lambda} \boldsymbol{e}=(1-\rho) \\
\times\left[\phi^{(D)} \sum_{n=0}^{N-1} \boldsymbol{\psi}_{n}\left[S^{*}(\theta)\right]^{n} \Xi\left(S^{*}(\theta)\right) \boldsymbol{D}_{g}\left\{\frac{\alpha_{i}\left(S^{*}(\theta)\right) H^{*}\left(\alpha_{i}\left(S^{*}(\theta)\right)\right)}{\theta-\alpha_{i}\left(S^{*}(\theta)\right)}\right\}_{i=1}^{m} \Xi^{-1}\left(S^{*}(\theta)\right)\right. \\
\left.+\phi^{(V)} \boldsymbol{\kappa} \Xi\left(S^{*}(\theta)\right) \boldsymbol{D}_{g}\left\{\frac{1-V^{*}\left(\alpha_{i}\left(S^{*}(\theta)\right)\right) H^{*}\left(\alpha_{i}\left(S^{*}(\theta)\right)\right)}{E(V)\left[\theta-\alpha_{i}\left(S^{*}(\theta)\right)\right]}\right\}_{i=1}^{m} \Xi^{-1}\left(S^{*}(\theta)\right)\right] \frac{\boldsymbol{D}}{\lambda} \boldsymbol{e}
\end{gathered}
$$

where $\boldsymbol{D} / \lambda$ is the factor that converts the virtual phase probabilities to actual phase probabilities.

Now, we obtain $W_{A}^{*(D)}(\theta)$. If the virtual customer arrives during the dormant period and sees $k$ customers, his waiting time is the sum of

(i) the service times of those $k$ customers,

(ii) the time until $(N-k-1)$ more customers arrive and

(iii) the set-up time.

The system is idle with probability $(1-\rho)$. Thus $(1-\rho) \phi^{(D)} \sum_{k=0}^{N-1} \boldsymbol{\psi}_{k} z^{k}$ is the

joint vector generating function of the number of customers that are found by the virtual customer who arrives during the dormant period. Using (2.48), we get

$$
W_{A}^{*(D)}(\theta)=(1-\rho) \phi^{(D)} \sum_{k=0}^{N-1} \boldsymbol{\psi}_{k}\left[S^{*}(\theta)\right]^{k} \frac{\boldsymbol{D}}{\lambda} \boldsymbol{T}_{N-k-1}^{*}(\theta) \boldsymbol{e} \boldsymbol{H}^{*}(\theta) .
$$

Now, we obtain $W_{A}^{*(V)}(\theta)$. Consider the virtual customer who arrives during a vacation. Suppose $k$ customers arrive during the elapsed vacation and $l$ customers arrive during the remaining vacation. Then we have two cases:

Case 1: If $k+l+1 \geq N$, the virtual waiting time is the sum of

(i) the remaining vacation time,

(ii) the $k$ service times and

(iii) the set-up time.

Case 2: If $k+l+1 \leq N-1$, the virtual waiting time is the sum of

(i) the remaining vacation time,

(ii) the time until $N-(k+l+1)$ more customers arrive,

(iii) the $k$ service times and

(iv) the set-up time.

Obtaining the LST of the actual waiting time due to $(i)$ and $(i i)$ of Case 1 and $(i)$ and $(i i i)$ of Case 2 requires the joint transform of the number of customers that arrive during the elapsed vacation time, the number of customers that arrive during the remaining vacation, and the length of the remaining vacation time. For this purpose, we employ the approach used in Kasahara, et al. [2], which is presented in Appendix II.

To compute the waiting time due to (ii) of Case 2, we can use (2.48). Then, the LST of the actual waiting time during the vacation can be expressed as

$$
\begin{gathered}
W_{A}^{*(V)}(\theta)=(1-\rho) \phi^{(V)} \boldsymbol{\kappa}\left\{\sum_{k=0}^{\infty} \sum_{l=\max (N-k-1,0)}^{\infty} \boldsymbol{\Omega}_{k, l}^{*}(\theta)\left[S^{*}(\theta)\right]^{k}\right. \\
\left.+\sum_{k=0}^{N-2} \sum_{l=0}^{N-k-2} \boldsymbol{\Omega}_{k, l}^{*}(\theta)\left[S^{*}(\theta)\right]^{k} \boldsymbol{T}_{N-k-l-1}^{*}(\theta)\right\} \boldsymbol{e} \boldsymbol{H}^{*}(\theta)
\end{gathered}
$$




$$
\begin{gathered}
=(1-\rho) \phi^{(V)} \boldsymbol{\kappa}\left\{\sum_{k=0}^{\infty} \sum_{l=0}^{\infty} \boldsymbol{\Omega}_{k, l}^{*}(\theta)\left[S^{*}(\theta)\right]^{k}\right. \\
\left.+\sum_{k=0}^{N-2} \sum_{l=0}^{N-k-2} \boldsymbol{\Omega}_{k, l}^{*}(\theta)\left[S^{*}(\theta)\right]^{k}\left[\boldsymbol{T}_{N-k-l-1}^{*}(\theta)-\boldsymbol{I}\right]\right\} \boldsymbol{e} \boldsymbol{H}^{*}(\theta) .
\end{gathered}
$$

To simplify the term $\sum_{k=0}^{\infty} \sum_{l=0}^{\infty}\left[S^{*}(\theta)\right]^{k} \boldsymbol{\Omega}_{k, l}^{*}(\theta) \boldsymbol{e}$ contained in (3.4), let $\omega_{i}^{*}(z, \theta)$ be

the joint transform of the number of customers that arrive during the elapsed vacation (based on the actual arrival point) and the length of the remaining vacation time with the phase at the start of the vacation being in $i$. Let its column vector be $\boldsymbol{\omega}^{*}(z, \theta)$. Then, from (II- 1 ) of Appendix II, we have

$$
\begin{gathered}
\boldsymbol{\omega}^{*}(z, \theta)=\left.\boldsymbol{\Omega}^{*}\left(z_{1}, z_{2}, \theta\right) \boldsymbol{e}\right|_{z_{1}=z, z_{2}=1} \\
=\int_{x=0}^{\infty} \int_{t=0}^{x} e^{(C+z D) t} \frac{\boldsymbol{D}}{\lambda}\left[\boldsymbol{I}+\sum_{n=1}^{\infty} \frac{(x-t)^{n}}{n !}(\boldsymbol{C}+\boldsymbol{D})^{n}\right] \boldsymbol{e} e^{-\theta(x-t)} \frac{v(x)}{E(V)} d t d x .
\end{gathered}
$$

From $(\boldsymbol{C}+\boldsymbol{D})^{n} \boldsymbol{e}=\mathbf{0},(n \geq 1)$ and (I-3) of Appendix I, (3.5a) is reduced to

$$
\boldsymbol{\omega}^{*}(z, \theta)=\Xi(z) \boldsymbol{D}_{g}\left\{\frac{V^{*}\left(\alpha_{i}(z)\right)-V^{*}(\theta)}{E(V)\left[\theta-\alpha_{i}(z)\right]}\right\}_{i=1}^{m} \Xi^{-1}(z) \frac{D}{\lambda} \boldsymbol{e} .
$$

Then, we have

$$
\begin{gathered}
\sum_{k=0}^{\infty} \sum_{l=0}^{\infty}\left[S^{*}(\theta)\right]^{k} \boldsymbol{\Omega}_{k, l}^{*}(\theta) \boldsymbol{e}=\boldsymbol{\omega}^{*}\left(S^{*}(\theta), \theta\right) \\
=\Xi\left(S^{*}(\theta)\right) \boldsymbol{D}_{g}\left\{\frac{V^{*}\left(\alpha_{i}\left(S^{*}(\theta)\right)\right)-V^{*}(\theta)}{E(V)\left[\theta-\alpha_{i}\left(S^{*}(\theta)\right)\right]}\right\}_{i=1}^{m} \Xi^{-1}\left(S^{*}(\theta)\right) \frac{\boldsymbol{D}}{\lambda} \boldsymbol{e} .
\end{gathered}
$$

Using (3.6) in (3.4), we get

$$
\begin{aligned}
W_{A}^{*(V)}(\theta)= & (1-\rho) \phi^{(V)} \boldsymbol{\kappa}\left\{\Xi\left(S^{*}(\theta)\right) \boldsymbol{D}_{g}\left\{\frac{V^{*}\left(\alpha_{i}\left(S^{*}(\theta)\right)\right)-V^{*}(\theta)}{E(V)\left[\theta-\alpha_{i}\left(S^{*}(\theta)\right)\right]}\right\}_{i=1}^{m} \Xi^{-1}\left(S^{*}(\theta)\right) \frac{D}{\lambda} \boldsymbol{e}\right. \\
& \left.+\sum_{k=0}^{N-2 N-k-2} \sum_{l=0}^{N} \boldsymbol{\Omega}_{k, l}^{*}(\theta)\left[S^{*}(\theta)\right]^{k}\left[\boldsymbol{T}_{N-k-l-1}^{*}(\theta)-\boldsymbol{I}\right] \boldsymbol{e}\right\} H^{*}(\theta) .
\end{aligned}
$$

Now, we obtain $W_{A}^{*(S)}(\theta)$. The virtual waiting time in this case is the sum of

(i) the remaining set-up time and

(ii) the service times of the waiting customers.

We can use (2.23) for this purpose. Using (2.19d), (2.28), (2.30), (2.38), and $(\theta \boldsymbol{I}+\boldsymbol{C}+z \boldsymbol{D})^{-1}=\Xi(z) \boldsymbol{D}_{g}\left\{\frac{1}{\theta-\alpha_{i}(z)}\right\}_{i=1}^{m} \Xi^{-1}(z)$, Equation (2.23) becomes 


$$
\begin{aligned}
\boldsymbol{p}^{*(S)}(z, \theta) & =(1-\rho)\left[\phi^{(D)} \sum_{n=0}^{N-1} \boldsymbol{\psi}_{n} z^{n} \Xi(z) \boldsymbol{D}_{g}\left\{\frac{\alpha_{i}(z)}{\theta-\alpha_{i}(z)}\right\}_{i=1}^{m} \Xi^{-1}(z)\right. \\
& \left.-\phi^{(V)} \boldsymbol{\kappa} \Xi(z) \boldsymbol{D}_{g}\left\{\frac{V^{*}\left(\alpha_{i}(z)\right)}{E(V)\left[\theta-\alpha_{i}(z)\right]}\right\}_{i=1}^{m} \Xi^{-1}(z)\right] \\
& \times \Xi(z) \boldsymbol{D}_{g}\left\{H^{*}(\theta)-H^{*}\left(\alpha_{i}(z)\right)\right\}_{i=1}^{m} \Xi^{-1}(z) .
\end{aligned}
$$

Thus, we get

$$
\begin{gathered}
W_{A}^{*(S)}(\theta)=\left.\boldsymbol{p}^{*(S)}(z, \theta)\right|_{z=S^{*}(\theta)} \frac{\boldsymbol{D}}{\lambda} \boldsymbol{e}=(1-\rho) \\
\times\left[\phi^{(D)} \sum_{n=0}^{N-1} \boldsymbol{\psi}_{n}\left[S^{*}(\theta)\right]^{n} \Xi\left(S^{*}(\theta)\right) \boldsymbol{D}_{g}\left\{\frac{\alpha_{i}\left(S^{*}(\theta)\right)}{\theta-\alpha_{i}\left(S^{*}(\theta)\right)}\right\}_{i=1}^{m} \Xi^{-1}\left(S^{*}(\theta)\right)\right. \\
\left.-\phi^{(V)} \boldsymbol{\kappa} \Xi\left(S^{*}(\theta)\right) \boldsymbol{D}_{g}\left\{\frac{V^{*}\left(\alpha_{i}\left(S^{*}(\theta)\right)\right)}{E(V)\left[\theta-\alpha_{i}\left(S^{*}(\theta)\right)\right]}\right\}_{i=1}^{m} \Xi^{-1}\left(S^{*}(\theta)\right)\right] \\
\times \Xi\left(S^{*}(\theta)\right) \boldsymbol{D}_{g}\left\{H^{*}(\theta)-H^{*}\left(\alpha_{i}\left(S^{*}(\theta)\right)\right)\right\}_{i=1}^{m} \Xi^{-1}\left(S^{*}(\theta)\right) \frac{\boldsymbol{D}}{\lambda} \boldsymbol{e} .
\end{gathered}
$$

Then, using (3.2), (3.3), (3.7) and (3.9), we get the LST of the actual waiting time as

$$
\begin{gathered}
W_{A}^{*}(\theta)=W_{A}^{*(V)}(\theta)+W_{A}^{*(D)}(\theta)+W_{A}^{*(S)}(\theta)+W_{A}^{*(B)}(\theta) \\
=\left\{\phi^{(D)} \sum_{k=0}^{N-1} \boldsymbol{\psi}_{k}\left[S^{*}(\theta)\right]^{k} H^{*}(\theta)+\phi^{(V)} \boldsymbol{\kappa}\left[\frac{1-V^{*}(\theta)}{E(V) \theta}\right] H^{*}(\theta)+\phi^{(S)} \boldsymbol{\kappa}\left[\frac{1-H^{*}(\theta)}{E(H) \theta}\right]\right\} \\
\times \Xi\left(S^{*}(\theta)\right) \boldsymbol{D}_{g}\left\{\frac{(1-\rho) \theta}{\theta-\alpha_{i}\left(S^{*}(\theta)\right)}\right\}_{i=1}^{m} \Xi^{-1}\left(S^{*}(\theta)\right) \frac{\boldsymbol{D}}{\lambda} \boldsymbol{e} \\
+(1-\rho) \phi^{(D)} \sum_{k=0}^{N-1} \boldsymbol{\psi}_{k}\left[S^{*}(\theta)\right]^{k} \frac{\boldsymbol{D}}{\lambda}\left[\boldsymbol{T}_{N-k-1}^{*}(\theta)-\boldsymbol{I}\right] \boldsymbol{e} H^{*}(\theta) \\
+(1-\rho) \phi^{(V)} \boldsymbol{\kappa} \sum_{k=0}^{N-2} \sum_{l=0}^{N-k-2}\left[S^{*}(\theta)\right]^{k} \boldsymbol{\Omega}_{k, l}^{*}(\theta)\left[\boldsymbol{T}_{N-k-l-1}^{*}(\theta)-\boldsymbol{I}\right] \boldsymbol{e} H^{*}(\theta)
\end{gathered}
$$

or, in matrix-analytic notations,

$$
W_{A}^{*}(\theta)=\left\{\phi^{(D)} \sum_{k=0}^{N-1} \boldsymbol{\psi}_{k}\left[S^{*}(\theta)\right]^{k} H^{*}(\theta)+\phi^{(V)} \boldsymbol{\kappa}\left[\frac{1-V^{*}(\theta)}{E(V) \theta}\right] H^{*}(\theta)+\phi^{(S)} \boldsymbol{\kappa}\left[\frac{1-H^{*}(\theta)}{E(H) \theta}\right]\right\}
$$




$$
\begin{gathered}
\times(1-\rho) \theta\left[\theta \boldsymbol{I}+\boldsymbol{C}+S^{*}(\theta) \boldsymbol{D}\right]^{-1} \frac{\boldsymbol{D}}{\lambda} \boldsymbol{e} \\
+(1-\rho) \phi^{(D)} \sum_{k=0}^{N-1} \boldsymbol{\psi}_{k}\left[S^{*}(\theta)\right]^{k} \frac{\boldsymbol{D}}{\lambda}\left[\boldsymbol{T}_{N-k-1}^{*}(\theta)-\boldsymbol{I}\right] \boldsymbol{e} H^{*}(\theta) \\
+(1-\rho) \phi^{(V)} \underset{\kappa}{\boldsymbol{\kappa} \sum_{k=0}^{N-2}} \sum_{l=0}^{N-k-2}\left[S^{*}(\theta)\right]^{k} \boldsymbol{\Omega}_{k, l}^{*}(\theta)\left[\boldsymbol{T}_{N-k-l-1}^{*}(\theta)-\boldsymbol{I}\right] \boldsymbol{e} H^{*}(\theta) .
\end{gathered}
$$

\section{Mean Performance Measures}

In this section, we derive the algorithm to compute the mean queue length and the mean waiting time.

\subsection{The Mean Queue Length}

We can get the mean queue length $L_{D}=\boldsymbol{X}^{(1)}(1) \boldsymbol{e}$ at departures by following the standard procedure presented in Lucantoni, et al. [10]. We first rewrite (2.46) as

$$
\begin{aligned}
& \boldsymbol{X}(z)[z \boldsymbol{I}-\boldsymbol{A}(z)]=\frac{1-\rho}{\lambda}\left\{\phi^{(D)} \sum_{n=0}^{N-1} \boldsymbol{\psi}_{n} z^{n}(\boldsymbol{C}+z \boldsymbol{D})+\frac{1}{E(V)} \phi^{(V)} \boldsymbol{\kappa}[\boldsymbol{V}(z)-\boldsymbol{I}]\right. \\
& \left.+\frac{1}{E(H)} \phi^{(S)} \boldsymbol{\kappa}\left[\sum_{n=0}^{N-1} \boldsymbol{\Phi}_{n}(-\boldsymbol{C})^{-1} z^{n}(\boldsymbol{C}+z \boldsymbol{D})+\boldsymbol{V}(z)\right][\boldsymbol{H}(z)-\boldsymbol{I}]\right\} \boldsymbol{A}(z) .
\end{aligned}
$$

Using $z=1$ in (4.1) and adding $\boldsymbol{X}(1) \boldsymbol{e} \boldsymbol{\pi}$ to both sides, we get

$$
\begin{gathered}
\boldsymbol{X}(1)=\boldsymbol{\pi}+\frac{1-\rho}{\lambda}\left\{\phi^{(D)} \sum_{n=0}^{N-1} \boldsymbol{\psi}_{n}(\boldsymbol{C}+\boldsymbol{D})+\frac{1}{E(V)} \phi^{(V)} \boldsymbol{\kappa}(\boldsymbol{V}-\boldsymbol{I})\right. \\
\left.+\frac{1}{E(H)} \phi^{(S)} \boldsymbol{\kappa}\left[\sum_{n=0}^{N-1} \boldsymbol{\Phi}_{n}(-\boldsymbol{C})^{-1}(\boldsymbol{C}+\boldsymbol{D})+\boldsymbol{V}\right](\boldsymbol{H}-\boldsymbol{I})\right\} \\
\times \boldsymbol{A}(\boldsymbol{I}-\boldsymbol{A}+\boldsymbol{e} \boldsymbol{\pi})^{-1}
\end{gathered}
$$

Let $\boldsymbol{U}(z)$ be the right-hand side of (4.1). Then, we get

$$
\begin{gathered}
\boldsymbol{U}^{(1)}(1)=\frac{1-\rho}{\lambda}\left\{\phi^{(D)} \sum_{n=0}^{N-1} n \boldsymbol{\psi}_{n}(\boldsymbol{C}+\boldsymbol{D})+\phi^{(D)} \sum_{n=0}^{N-1} \boldsymbol{\psi}_{n} \boldsymbol{D}\right. \\
+\frac{1}{E(V)} \phi^{(V)} \boldsymbol{\kappa} \boldsymbol{V}^{(1)}(1)
\end{gathered}
$$




$$
\begin{gathered}
+\frac{1}{E(H)} \phi^{(S)} \boldsymbol{\kappa}\left[\sum_{n=0}^{N-1} n \boldsymbol{\Phi}_{n}(-\boldsymbol{C})^{-1}(\boldsymbol{C}+\boldsymbol{D})+\sum_{n=0}^{N-1} \boldsymbol{\Phi}_{n}(-\boldsymbol{C})^{-1} \boldsymbol{D}+\boldsymbol{V}^{(1)}(1)\right](\boldsymbol{H}-\boldsymbol{I}) \\
\left.+\frac{1}{E(H)} \phi^{(S)} \boldsymbol{\kappa}\left[\sum_{n=0}^{N-1} \boldsymbol{\Phi}_{n}(-\boldsymbol{C})^{-1}(\boldsymbol{C}+\boldsymbol{D})+\boldsymbol{V}\right] \boldsymbol{H}^{(1)}(a)\right\} \boldsymbol{A} \\
+\frac{1-\rho}{\lambda}\left\{\phi^{(D)} \sum_{n=0}^{N-1} \boldsymbol{\psi}_{n}(\boldsymbol{C}+\boldsymbol{D})+\frac{1}{E(V)} \phi^{(V)} \boldsymbol{\kappa}(\boldsymbol{V}-\boldsymbol{I})\right. \\
\left.+\frac{1}{E(H)} \phi^{(S)} \boldsymbol{\kappa}\left[\sum_{n=0}^{N-1} \boldsymbol{\Phi}_{n}(-\boldsymbol{C})^{-1}(\boldsymbol{C}+\boldsymbol{D})+\boldsymbol{V}\right](\boldsymbol{H}-\boldsymbol{I})\right\} \boldsymbol{A}^{(1)}(1)
\end{gathered}
$$

and

$$
\begin{aligned}
& \boldsymbol{U}^{(2)}(1) \boldsymbol{e}=\frac{1-\rho}{\lambda}\left\{2 \phi^{(D)} \sum_{n=0}^{N-1} n \boldsymbol{\psi}_{n} \boldsymbol{D} \boldsymbol{e}+\frac{1}{E(V)} \phi^{(V)} \boldsymbol{\kappa} \boldsymbol{V}^{(2)}(1) \boldsymbol{e}\right. \\
& +\frac{2}{E(H)} \phi^{(S)} \boldsymbol{\kappa}\left[\sum_{n=0}^{N-1} n \boldsymbol{\Phi}_{n}(-\boldsymbol{C})^{-1}(\boldsymbol{C}+\boldsymbol{D})+\sum_{n=0}^{N-1} \boldsymbol{\Phi}_{n}(-\boldsymbol{C})^{-1} \boldsymbol{D}+\boldsymbol{V}^{(1)}(1)\right] \boldsymbol{H}^{(1)}(1) \boldsymbol{e} \\
& \left.+\frac{1}{E(H)} \phi^{(S)} \boldsymbol{\kappa}\left[\sum_{n=0}^{N-1} \boldsymbol{\Phi}_{n}(-\boldsymbol{C})^{-1}(\boldsymbol{C}+\boldsymbol{D})+\boldsymbol{V}\right] \boldsymbol{H}^{(1)}(1) \boldsymbol{e}\right\} \\
& +\frac{2(1-\rho)}{\lambda}\left\{\phi^{(D)} \sum_{n=0}^{N-1} n \boldsymbol{\psi}_{n}(\boldsymbol{C}+\boldsymbol{D})+\phi^{(D)} \sum_{n=0}^{N-1} \boldsymbol{\psi}_{n} \boldsymbol{D}\right. \\
& +\frac{1}{E(V)} \phi^{(V)} \boldsymbol{\kappa} \boldsymbol{V}^{(1)}(1) \\
& +\frac{1}{E(H)} \phi^{(S)} \boldsymbol{\kappa}\left[\sum_{n=0}^{N-1} n \boldsymbol{\Phi}_{n}(-\boldsymbol{C})^{-1}(\boldsymbol{C}+\boldsymbol{D})+\sum_{n=0}^{N-1} \boldsymbol{\Phi}_{n}(-\boldsymbol{C})^{-1} \boldsymbol{D}+\boldsymbol{V}^{(1)}(1)\right](\boldsymbol{H}-\boldsymbol{I}) \\
& \left.+\frac{1}{E(H)} \phi^{(S)} \boldsymbol{\kappa}\left[\sum_{n=0}^{N-1} \boldsymbol{\Phi}_{n}(-\boldsymbol{C})^{-1}(\boldsymbol{C}+\boldsymbol{D})+\boldsymbol{V}\right] \boldsymbol{H}^{(1)}(1)\right\} \boldsymbol{A}^{(1)}(1) \boldsymbol{e}
\end{aligned}
$$




$$
\begin{gathered}
+\frac{1-\rho}{\lambda}\left\{\phi^{(D)} \sum_{n=0}^{N} \boldsymbol{\psi}_{n}(\boldsymbol{C}+\boldsymbol{D})+\frac{1}{E(V)} \phi^{(V)} \boldsymbol{\kappa}(\boldsymbol{V}-\boldsymbol{I})\right. \\
\left.+\frac{1}{E(H)} \phi^{(S)} \boldsymbol{\kappa}\left[\sum_{n=0}^{N-1} \boldsymbol{\Phi}_{n}(-\boldsymbol{C})^{-1}(\boldsymbol{C}+\boldsymbol{D})+\boldsymbol{V}\right](\boldsymbol{H}-\boldsymbol{I})\right\} \boldsymbol{A}^{(2)}(1) \boldsymbol{e} .
\end{gathered}
$$

Then, we get the mean queue length at departures as

$$
\begin{gathered}
L_{D}=\boldsymbol{X}^{(1)}(1) \boldsymbol{e} \\
=\frac{1}{2(1-\rho)}\left\{\boldsymbol{X}(1) \boldsymbol{A}^{(2)}(1) \boldsymbol{e}+\boldsymbol{U}^{(2)}(1) \boldsymbol{e}\right. \\
\left.+2\left[\boldsymbol{U}^{(1)}(1)-\boldsymbol{X}(1)\left(\boldsymbol{I}-\boldsymbol{A}^{(1)}(1)\right)\right](\boldsymbol{I}-\boldsymbol{A}+\boldsymbol{e} \boldsymbol{\pi})^{-1} \boldsymbol{\beta}\right\} .
\end{gathered}
$$

Then, the mean queue length at an arbitrary time can be obtained, from (2.47), as

$$
\begin{gathered}
L=\boldsymbol{Y}^{(1)}(1) \boldsymbol{e} \\
=L_{D}+\left[\frac{1}{\lambda} \boldsymbol{\pi} \boldsymbol{D}-\boldsymbol{X}(1)\right](\boldsymbol{e} \boldsymbol{\pi}+\boldsymbol{C}+\boldsymbol{D})^{-1} \boldsymbol{D} \boldsymbol{e} .
\end{gathered}
$$

\subsection{The Mean Waiting Time}

We rewrite (3.10b) as

$$
\begin{gathered}
W_{A}^{*}(\theta)=(1-\rho) \phi^{(D)} \sum_{k=0}^{N-1} \boldsymbol{\psi}_{k}\left[S^{*}(\theta)\right]^{k} H^{*}(\theta) \frac{\boldsymbol{D}}{\lambda}\left[\boldsymbol{T}_{N-k-1}^{*}(\theta)-\boldsymbol{I}\right] \boldsymbol{e} \\
+(1-\rho) \phi^{(V)} \sum_{k=0}^{N-2} \sum_{l=0}^{N-k-2}\left[S^{*}(\theta)\right]^{k} \boldsymbol{\Omega}_{k, l}^{*}(\theta) H^{*}(\theta)\left[\boldsymbol{T}_{N-k-l-1}^{*}(\theta)-\boldsymbol{I}\right] \boldsymbol{e} \\
+\widetilde{\boldsymbol{W}}_{N, \text { setup }}^{*}(\theta) \frac{\boldsymbol{D}}{\lambda} \boldsymbol{e}
\end{gathered}
$$

where

$$
\begin{gathered}
\widetilde{\boldsymbol{W}}_{N, \text { setup }}^{*}(\theta)=\left\{\phi^{(D)} \sum_{k=0}^{N-1} \boldsymbol{\psi}_{k}\left[S^{*}(\theta)\right]^{k} H^{*}(\theta)+\phi^{(V)} \boldsymbol{\kappa}\left[\frac{1-V^{*}(\theta)}{E(V) \theta}\right] H^{*}(\theta)\right. \\
\left.+\phi^{(S)} \boldsymbol{\kappa}\left[\frac{1-H^{*}(\theta)}{E(H) \theta}\right]\right\}(1-\rho) \theta\left[\theta \boldsymbol{I}+\boldsymbol{C}+S^{*}(\theta) \boldsymbol{D}\right]^{-1} .
\end{gathered}
$$

Then the mean waiting tie can be obtained from 


$$
\begin{gathered}
W_{A}=-W_{A}^{*(1)}(0)=(1-\rho)\left[\phi^{(D)} \sum_{k=0}^{N-1} \boldsymbol{\psi}_{k} \frac{\boldsymbol{D}}{\lambda} \boldsymbol{\tau}_{N-k-1}+\phi^{(V)} \boldsymbol{\kappa} \sum_{k=0}^{N-2} \sum_{l=0}^{N-k-2} \boldsymbol{\Omega}_{k, l} \boldsymbol{\tau}_{N-k-l-1}\right] \\
-\widetilde{\boldsymbol{W}}_{N, \text { setup }}^{*(1)}(0) \frac{\boldsymbol{D}}{\lambda} \boldsymbol{e}
\end{gathered}
$$

where $\boldsymbol{\Omega}_{k, l}$ can be obtained from (II-6) of Appendix II and $\boldsymbol{\tau}_{n}$ can be obtained from (2.50).

We rewrite (4.8) as

$$
\begin{gathered}
\widetilde{W}_{N, \text { setup }}^{*}(\theta)\left[\theta \boldsymbol{I}+\boldsymbol{C}+S^{*}(\theta) \boldsymbol{D}\right]=(1-\rho)\left\{\phi^{(D)} \sum_{n=0}^{N-1} \boldsymbol{\psi}_{n}\left[S^{*}(\theta)\right]^{n} \theta H^{*}(\theta)\right. \\
\left.+\frac{1}{E(V)} \phi^{*(V)} \boldsymbol{\kappa}\left[1-V^{*}(\theta)\right] H^{*}(\theta)+\frac{1}{E(H)} \phi^{(S)} \boldsymbol{\kappa}\left[1-H^{*}(\theta)\right]\right\} .
\end{gathered}
$$

Then, we easily get

$$
\widetilde{\boldsymbol{W}}_{N}^{*}(0)=\boldsymbol{\pi}
$$

Differentiating (4.10) with respect to $\theta$, we get

$$
\begin{gathered}
\widetilde{\boldsymbol{W}}_{N, \text { setup }}^{*(1)}(\theta)\left[\theta \boldsymbol{I}+\boldsymbol{C}+S^{*}(\theta) \boldsymbol{D}\right]+\widetilde{\boldsymbol{W}}_{N, \text { setup }}^{*}(\theta)\left[\boldsymbol{I}+S^{*(1)}(\theta) \boldsymbol{D}\right] \\
=(1-\rho)\left\{\phi^{(D)} \sum_{n=0}^{N-1} \boldsymbol{\psi}_{n} n\left[S^{*}(\theta)\right]^{n-1} S^{*(1)}(\theta) \theta H^{*}(\theta)\right. \\
+\phi^{(D)} \sum_{n=0}^{N-1} \boldsymbol{\psi}_{n}\left[S^{*}(\theta)\right]^{n} H^{*}(\theta)+\phi^{(D)} \sum_{n=0}^{N-1} \boldsymbol{\psi}_{n}\left[S^{*}(\theta)\right]^{n} \theta H^{*(1)}(\theta) \\
-\frac{1}{E(V)} \phi^{(V)} \boldsymbol{\kappa} V^{*(1)}(\theta) H^{*}(\theta)+\frac{1}{E(V)} \boldsymbol{\kappa}\left[1-V^{*}(\theta)\right] H^{*(1)}(\theta) \\
\left.-\frac{1}{E(H)} \phi^{(S)} \boldsymbol{\kappa} H^{*(1)}(\theta)\right\}
\end{gathered}
$$

where $f^{(1)}(x)=\frac{d}{d x} f(x)$. Using $\theta=0$ in (4.12) and adding $\widetilde{\boldsymbol{W}}_{N}^{*(1)}(0) \boldsymbol{e} \boldsymbol{\pi}$ to both sides yields

$$
\begin{gathered}
\widetilde{\boldsymbol{W}}_{N, \text { setup }}^{*(1)}(0)=\left[\widetilde{\boldsymbol{W}}_{N, \text { setup }}^{*(1)}(0) \boldsymbol{e}\right] \boldsymbol{\pi} \\
+(1-\rho)\left\{\phi^{(D)} \sum_{n=0}^{N-1} \boldsymbol{\psi}_{n}+\left(\phi^{(V)}+\phi^{(S)}\right) \boldsymbol{\kappa}-\frac{1}{1-\rho} \boldsymbol{\pi}[\boldsymbol{I}-E(S) \boldsymbol{D}]\right\}(\boldsymbol{e} \boldsymbol{\pi}+\boldsymbol{C}+\boldsymbol{D})^{-1} .
\end{gathered}
$$

Postmultiplying (4.13) by $\boldsymbol{D e}$ yields 


$$
\begin{gathered}
\widetilde{\boldsymbol{W}}_{N, \text { setup }}^{*(1)}(0) \boldsymbol{D} \boldsymbol{e}=\lambda \widetilde{\boldsymbol{W}}_{N, \text { setup }}^{*(1)}(0) \boldsymbol{e} \\
+(1-\rho)\left\{\phi^{(D)} \sum_{n=0}^{N-1} \boldsymbol{\psi}_{n}+\left(\phi^{(V)}+\phi^{(S)}\right) \boldsymbol{\kappa}-\frac{1}{1-\rho} \boldsymbol{\pi}[\boldsymbol{I}-E(S) \boldsymbol{D}]\right\}(\boldsymbol{e} \boldsymbol{\pi}+\boldsymbol{C}+\boldsymbol{D})^{-1} \boldsymbol{D} \boldsymbol{e} .
\end{gathered}
$$

Differentiating (4.12) once more, using $\theta=0$ and postmultiplying $\boldsymbol{e}$ yields

$$
\begin{gathered}
\widetilde{\boldsymbol{W}}_{N, \text { setup }}^{*(1)}(0) \boldsymbol{e}=E(S) \widetilde{\boldsymbol{W}}_{N, \text { setup }}^{*(1)}(0) \boldsymbol{D} \boldsymbol{e}-\frac{1}{2} \lambda E\left(S^{2}\right) \\
-(1-\rho)\left\{\phi^{(D)}\left[E(S) \sum_{n=1}^{N-1} n \boldsymbol{\psi}_{n} \boldsymbol{e}+E(H)\right]+\phi^{(V)}\left[\frac{E\left(V^{2}\right)}{2 E(V)}+E(H)\right]+\phi^{(S)} \frac{E\left(H^{2}\right)}{2 E(H)}\right\} .
\end{gathered}
$$

From (4.14) and (4.15), we get

$$
\begin{gathered}
-\widetilde{\boldsymbol{W}}_{N, \text { setup }}^{*(1)}(0) \frac{\boldsymbol{D}}{\lambda} \boldsymbol{e}=\frac{\lambda E\left(S^{2}\right)}{2(1-\rho)}+\phi^{(D)}\left[E(S) \sum_{n=1}^{N-1} n \boldsymbol{\psi}_{n} \boldsymbol{e}+E(H)\right] \\
+\phi^{(V)}\left[\frac{E\left(V^{2}\right)}{2 E(V)} E(H)\right]+\phi^{(S)} \frac{E\left(H^{2}\right)}{2 E(H)} \\
+\left\{\frac{1}{1-\rho} \boldsymbol{\pi}[\boldsymbol{I}-E(S) \boldsymbol{D}]-\phi^{(D)} \sum_{n=0}^{N-1} \boldsymbol{\psi}_{n}-\left(\phi^{(V)}+\phi^{(S)}\right) \boldsymbol{\kappa}\right\}(\boldsymbol{e} \boldsymbol{\pi}+\boldsymbol{C}+\boldsymbol{D})^{-1} \frac{\boldsymbol{D}}{\lambda} \boldsymbol{e}
\end{gathered}
$$

We finally using (4.16) in (4.9) to obtain the mean waiting time as

$$
\begin{gathered}
W_{A}=\frac{\lambda E\left(S^{2}\right)}{2(1-\rho)}+\phi^{(D)}\left[E(S) \sum_{n=1}^{N-1} n \boldsymbol{\psi}_{n} \boldsymbol{e}+E(H)\right] \\
+\phi^{(V)}\left[\frac{E\left(V^{2}\right)}{2 E(V)}+E(H)\right]+\phi^{(S)} \frac{E\left(H^{2}\right)}{2 E(H)} \\
+(1-\rho) \phi^{(D)} \sum_{n=0}^{N-1} \boldsymbol{\psi}_{n} \frac{\boldsymbol{D}}{\lambda} \boldsymbol{\tau}_{N-n-1}+(1-\rho) \phi^{(V)} \boldsymbol{\kappa} \sum_{k=0}^{N-2 N \sum_{l=0}^{N-k-2}} \boldsymbol{\Omega}_{k, l} \boldsymbol{\tau}_{N-k-l-1} \\
+\left\{\frac{1}{1-\rho} \boldsymbol{\pi}[\boldsymbol{I}-E(S) \boldsymbol{D}]-\phi^{(D)} \sum_{n=0}^{N-1} \boldsymbol{\psi}_{n}-\left(\phi^{(V)}+\phi^{(S)}\right) \boldsymbol{\kappa}\right\}(\boldsymbol{e} \boldsymbol{\pi}+\boldsymbol{C}+\boldsymbol{D})^{-1} \frac{\boldsymbol{D}}{\lambda} \boldsymbol{e}
\end{gathered}
$$




\section{Special Cases}

Let us show some special cases.

\subsection{M/G/1 Queue with Single Vacation}

In this case, we have $\boldsymbol{C}=-\lambda, \boldsymbol{D}=\lambda, \quad N=1, \phi^{(S)}=0, H^{*}(\theta)=1, \boldsymbol{V}_{0}=V^{*}(\lambda)$, $\phi^{(V)}=\frac{\lambda E(V)}{V^{*}(\lambda)+\lambda E(V)}, \phi^{(D)}=\frac{V^{*}(\lambda)}{V^{*}(\lambda)+\lambda E(V)}, \phi_{0}=1, V^{+}(z)=\frac{1-V^{*}(\lambda-\lambda z)}{\lambda(1-z) E(V)}$.

Using these in (2.62), (2.63) and (3.10b), we get the well-known results,

$$
\begin{gathered}
\boldsymbol{Y}(z)=\boldsymbol{X}(z)=\left[\frac{\lambda E(V)}{V^{*}(\lambda)+\lambda E(V)} \frac{1-V^{*}(\lambda-\lambda z)}{\lambda(1-z) E(V)}+\frac{V^{*}(\lambda)}{V^{*}(\lambda)+\lambda E(V)}\right] \\
\times \frac{(1-\rho)(z-1) S^{*}(\lambda-\lambda z)}{z-S^{*}(\lambda-\lambda z)} \\
W_{A}^{*}(\theta)=\left[\frac{\lambda E(V)}{V^{*}(\lambda)+\lambda E(V)} \frac{1-V^{*}(\theta)}{\theta E(V)}+\frac{V^{*}(\lambda)}{V^{*}(\lambda)+\lambda E(V)}\right] \frac{\theta(1-\rho)}{\theta-\lambda+\lambda S^{*}(\theta)} .
\end{gathered}
$$

\subsection{MAP/G/1 Queue with a Single Vacation}

In this case, we have $\quad N=1, \quad \phi^{(S)}=0, \quad H^{*}(\theta)=1, \quad \phi^{(D)}=\frac{\kappa V_{0}(-C)^{-1} \boldsymbol{e}}{\kappa V_{0}(-C)^{-1} \boldsymbol{e}+E(V)}$, $\phi^{(V)}=\frac{E(V)}{\kappa V_{0}(-C)^{-1} e+E(V)}, \boldsymbol{\psi}_{0}=\frac{\kappa V_{0}(-C)^{-1}}{\kappa V_{0}(-C)^{-1} e}$ and $\boldsymbol{\psi}_{n}=\mathbf{0},(n \geq 1)$. Using these in (2.62), (2.63), (3.10b) and (4.17), we get

$$
\begin{aligned}
\boldsymbol{Y}(z)= & {\left[\frac{\boldsymbol{\kappa} V_{0}(-C)^{-1}}{\kappa V_{0}(-C)^{-1} \boldsymbol{e}+E(V)}+\frac{E(V)}{\kappa V_{0}(-C)^{-1} \boldsymbol{e}+E(V)} \boldsymbol{\kappa} \boldsymbol{V}^{+}(z)\right] } \\
& \times(1-\rho)(z-1) \boldsymbol{A}(z)[z \boldsymbol{I}-\boldsymbol{A}(z)]^{-1} \\
\boldsymbol{X}(z)= & {\left[\frac{\kappa V_{0}(-\boldsymbol{C})^{-1}}{\kappa V_{0}(-C)^{-1} \boldsymbol{e}+E(V)}+\frac{E(V)}{\kappa V_{0}(-\boldsymbol{C})^{-1} \boldsymbol{e}+E(V)} \boldsymbol{\kappa} \boldsymbol{V}^{+}(z)\right] } \\
\times & (1-\rho)(\boldsymbol{C}+z \boldsymbol{D}) \boldsymbol{A}(z)[z \boldsymbol{I}-\boldsymbol{A}(z)]^{-1} \\
W_{A}^{*}(\theta)= & {\left[\frac{\kappa V_{0}(-C)^{-1}}{\boldsymbol{\kappa} V_{0}(-C)^{-1} \boldsymbol{e}+E(V)}+\frac{E(V)}{\kappa V_{0}(-\boldsymbol{C})^{-1} \boldsymbol{e}+E(V)} \boldsymbol{\kappa} \frac{1-V^{*}(\theta)}{\theta E(V)}\right] } \\
& \times(1-\rho) \theta\left[\theta \boldsymbol{I}+\boldsymbol{C}+S^{*}(\theta) \boldsymbol{D}\right]^{-1} \frac{D}{\lambda} \boldsymbol{e} \\
& \quad W_{A}=\frac{\lambda E\left(S^{2}\right)}{2(1-\rho)}+\phi^{(V)} \frac{E\left(V^{2}\right)}{2 E(V)}
\end{aligned}
$$




$$
+\left\{\frac{1}{1-\rho} \boldsymbol{\pi}[\boldsymbol{I}-E(S) \boldsymbol{D}]-\phi^{(D)} \boldsymbol{\psi}_{0}-\phi^{(V)} \boldsymbol{\kappa}\right\}(\boldsymbol{e} \boldsymbol{\pi}+\boldsymbol{C}+\boldsymbol{D})^{-1} \frac{\boldsymbol{D}}{\lambda} \boldsymbol{e}
$$

\subsection{MAP/G/1 Queue with a Single Vacation and N-Policy}

The results of this case can be obtained by simply using $\phi^{(S)}=0$ and $H^{*}(\theta)=1$ in (2.62), (2.63), (3.10b) and (4.17).

\section{References}

[1] Hwang, G.U., The MAP/G/1 Queue and its Applications to ATM Networks, Ph.D. Thesis, KAIST, Dept. of Mathematics, Korea 1997.

[2] Kasahara, S., Takine, T., Takahashi, Y., and Hasegawa, T., AP/G/1 queues under Npolicy with and without vacations, J. OR. Soc. Japan 39:2 (1996), 188-212.

[3] Kasahara, S., Takine, T., Takahashi, Y. and Hasegawa, Y., Analysis of an SPP/G/1 system with multiple vacations and E-limited service discipline, Queueing Sys. 14 (1993), 349-367.

[4] Lee, H.W., Moon, J.M., and Park, J.G., An analysis of the BMAP/G/1 queue I: Continuous time case, (1999), submitted.

[5] Lee, H.W. and Ahn, B.Y., Decompositions of the queue length distributions of the MAP/G/1 queue under multiple and single vacations with N-policy, (1999), submitted.

[6] Lee, H.W. and Ahn, B.Y., Analysis of the MAP/G/1 queue with multiple vacations, Npolicy and setup, (1999), submitted.

[7] Lee, H.W., Lee, S.S., Park, J.O., and Chae, K.C., Analysis of $\mathrm{M}^{\mathrm{x}} / \mathrm{G} / 1$ queue with Npolicy and multiple vacations, J. Appl. Prob. 31 (1994), 476-496.

[8] Lucantoni, D.M., New results on the single server queue with BMAP, Stoch. Models 7:1 (1991), 1-46.

[9] Lucantoni, D.M., The BMAP/G/1 queue: A tutorial, In: Models and Techniques for Performance Evaluation of Computer and Communications Systems (ed. by L. Donatiello and R. Nelson), Springer-Verlag (1993), 330-358.

[10] Lucantoni, D.M., Meier-Hellstern, K., and Neuts, M.F., A single server queue with server vacations and a class of non-renewal arrival process, Adv. Appl. Prob. 22 (1990), 676-705.

[11] Neuts, M.F., A versatile Markovian point process, J. Appl. Prob. 16 (1979), 764-779.

[12] Neuts, M.F., Structured Stochastic Matrices of M/G/1 Type and Their Applications, Marcel Dekker, New York 1989.

[13] Niu, Z., Takahashi, Y., and Endo, N., Performance evaluation of SVC-based IP-overATM networks, IEICE Trans. Commun. E81-B:5 (1998), 948-957.

[14] Niu, Z.. and Takahashi, Y., A finite-capacity queue with exhaustive vacation/closedown/setup times and Markovian arrival processes, Queueing Sys. 31 (1999), 1-23.

[15] Ramaswami, V., Th N/G/1 queue and its detailed analysis, Adv. Appl. Prob. 12 (1980), 222-261.

[16] Ramaswami, V., Stable recursion for the steady state vector for Markov chains of M/G/1 type, Stoch. Models 4 (1988), 183-188.

[17] Strang, G., Linear Algebra and its Applications, 2nd ed., Academic Press, New York 1980. 
[18] Takine, T. and Takahashi, Y., On the relationship between queue length at a random time and at a departure in the stationary queue with BMAP arrivals, Stoch. Models 14;3 (1998), 601-610.

\section{Appendix I: Representations in Matrix-Analytic Notations}

Since $a_{1}(z), \ldots, a_{m}(z)$ are eigenvectors of the matrix $-(\boldsymbol{C}+z \boldsymbol{D})$, we have (Strang [17])

$$
\begin{gathered}
-(\boldsymbol{C}+z \boldsymbol{D})=\Xi(z) \boldsymbol{D}_{g}\left\{\alpha_{i}(z)\right\}_{i=1}^{m} \Xi^{-1}(z), \\
-(\boldsymbol{C}+z \boldsymbol{D})^{-1}=\Xi(z) \boldsymbol{D}_{g}\left\{\frac{1}{\alpha_{i}(z)}\right\}_{i=1}^{m} \Xi^{-1}(z),
\end{gathered}
$$

and

$$
e^{(\boldsymbol{C}+\boldsymbol{D} z) x}=\sum_{n=0}^{\infty} \frac{[(\boldsymbol{C}+z \boldsymbol{D}) x]^{n}}{n !}=\Xi(z) \boldsymbol{D}_{g}\left\{e^{-\alpha_{i}(z) x}\right\}_{i=1}^{m} \Xi^{-1}(z)
$$

Let $\boldsymbol{A}(z)$ be the matrix generating function of the number of customers that arrive during a service time. Then, we have (Lucantoni, et al. [10])

$$
\boldsymbol{A}(z)=\int_{0}^{\infty} e^{(\boldsymbol{C}+z \boldsymbol{D}) x} d S(x)
$$

Using (I-3), we get the identity

$$
\boldsymbol{A}(z)=\Xi(z) \boldsymbol{D}_{g}\left\{S^{*}\left(\alpha_{i}(z)\right)\right\}_{i=1}^{m} \Xi^{-1}(z) .
$$

Then we get

$$
[z \boldsymbol{I}-\boldsymbol{A}(z)]^{-1}=\Xi(z) \boldsymbol{D}_{g}\left\{\frac{1}{z-S^{*}\left(\alpha_{i}(z)\right)}\right\}_{i=1}^{m} \Xi^{-1}(z) .
$$

Similarly, let $\boldsymbol{V}(z)$ be the matrix generating function of the number of customers that arrive during a vacation time. Then, analogously with (I-5), we get

$$
\boldsymbol{V}(z)=\Xi(z) \boldsymbol{D}_{g}\left\{V^{*}\left(\alpha_{i}(z)\right)\right\}_{i=1}^{m} \Xi^{-1}(z) .
$$

For later use, let $\boldsymbol{V}^{+}(z)$ be the matrix generating function of the number of customers that arrive during an elapsed (or remaining) vacation time. Then, after using (I-3), we obtain

$$
\begin{aligned}
\boldsymbol{V}^{+}(z) & =\int_{0}^{\infty} e^{(\boldsymbol{C}+z \boldsymbol{D}) x}\left[\frac{1-V(x)}{E(V)}\right] d x \\
& =\int_{0}^{\infty} \Xi(z) \boldsymbol{D}_{g}\left\{e^{-\alpha_{i}(z) x}\right\}_{i=1}^{m} \Xi^{-1}(z) \frac{1-V(x)}{E(V)} d x
\end{aligned}
$$




$$
\begin{gathered}
=\Xi(z) \boldsymbol{D}_{g}\left\{\int_{0}^{\infty} e^{-\alpha_{i}(z) x} \frac{1-V(x)}{E(V)} d x\right\}_{i=1}^{m} \Xi^{-1}(z) \\
=\Xi(z) \boldsymbol{D}_{g} \quad\left\{\frac{1-V^{*}\left(\alpha_{i}(z)\right)}{E(V) \alpha_{i}(z)}\right\}_{i=1}^{m} \Xi^{-1}(z) .
\end{gathered}
$$

We note that $\boldsymbol{A}(z), \boldsymbol{V}(z),(\boldsymbol{C}+z \boldsymbol{D})$ and $\boldsymbol{V}^{+}(z)$ commute with each other. From (I-5) and (I-7), (I-8) becomes, in matrix-analytic notations,

$$
\begin{aligned}
\boldsymbol{V}^{+}(z) & =\frac{1}{E(V)}\left[\Xi(z) \boldsymbol{I} \Xi^{-1}(z)-\Xi(z) \boldsymbol{D}_{g}\left\{V^{*}\left(\alpha_{i}(z)\right)\right\}_{i=1}^{m} \Xi^{-1}(z)\right] \\
& \times \Xi(z) \boldsymbol{D}_{g}\left\{\frac{1}{\alpha_{i}(z)}\right\}_{i=1}^{m} \Xi^{-1}(z) \\
& =\frac{[\boldsymbol{V}(z)-\boldsymbol{I}](\boldsymbol{C}+z \boldsymbol{D})^{-1}}{E(V)} \\
& =\frac{(\boldsymbol{C}+z \boldsymbol{D})^{-1}[\boldsymbol{V}(z)-\boldsymbol{I}]}{E(V)}
\end{aligned}
$$

Likewise, for the setup time $H$, we have

$$
\boldsymbol{H}(z)=\Xi(z) \boldsymbol{D}_{g}\left\{H^{*}\left(\alpha_{i}(z)\right)\right\}_{i=1}^{m} \Xi^{-1}(z)
$$

and

$$
\begin{gathered}
\boldsymbol{H}^{+}(z)=\Xi(z) \boldsymbol{D}_{g}\left\{\frac{1-H^{*}\left(\alpha_{i}(z)\right)}{E(H) \alpha_{i}(z)}\right\}_{i=1}^{m} \Xi^{-1}(z) \\
=\frac{[\boldsymbol{H}(z)-\boldsymbol{I}](\boldsymbol{C}+z \boldsymbol{D})^{-1}}{E(H)}
\end{gathered}
$$

\section{Appendix II The Approach of Kasahara, et al. [2]}

Let us define $\left(\boldsymbol{\Omega}_{k, l}(y)\right)_{i j}$ as the probability that, given the phase being in $i$ at the beginning of the vacation and an actual customer arrival during the vacation, $k$ customers arrive during the elapsed vacation time, $l$ customers arrive during the remaining vacation time, the remaining vacation time is less than or equal to $y$ and the phase is $j$ at the end of the vacation. Let us define the joint matrix transform $\Omega^{*}\left(z_{1}, z_{2}, \theta\right)$ as

$$
\boldsymbol{\Omega}^{*}\left(z_{1}, z_{2}, \theta\right) \quad=\int_{0}^{\infty} \sum_{k=0}^{\infty} \sum_{l=0}^{\infty} z_{1}^{k} z_{2}^{l} e^{-\theta y} d \boldsymbol{\Omega}_{k l}(y)
$$




$$
=\int_{x=0}^{\infty} \int_{t=0}^{x} e^{\left(\boldsymbol{C}+z_{1} \boldsymbol{D}\right) t} \frac{\boldsymbol{D}}{\lambda} e^{\left(\boldsymbol{C}+z_{2} \boldsymbol{D}\right)(x-t)} e^{-\theta(x-t)} \frac{1}{x} \frac{x v(x)}{E(V)} d t d x .
$$

Kasahara et al. [2] showed that

$$
\begin{aligned}
\boldsymbol{\Omega}^{*}\left(z_{1}, z_{2}, \theta\right) & =\int_{x=0}^{\infty} \int_{t=0}^{x} e^{\theta t} e^{-(\Theta+\theta) x} \cdot \frac{v(x)}{E(V)} \\
& \times \sum_{n=0}^{\infty} \sum_{m=0}^{\infty} \frac{t^{n}(x-t)^{m}}{n ! m !}\left(\Theta \boldsymbol{I}+\boldsymbol{C}+z_{1} \boldsymbol{D}\right)^{n} \frac{\boldsymbol{D}}{\lambda}\left(\Theta \boldsymbol{I}+\boldsymbol{C}+z_{2} \boldsymbol{D}\right)^{m} d t d x
\end{aligned}
$$

where $\Theta=\max _{i}(-C)_{i i}$.

They also showed that the following recursions hold

$$
\begin{aligned}
& \boldsymbol{F}_{k+1, l}(n, m)=\left\{\begin{array}{cl}
(\Theta \boldsymbol{I}+\boldsymbol{C}) \boldsymbol{F}_{k, l}(0, m), & (n=0) \\
(\Theta \boldsymbol{I}+\boldsymbol{C}) \boldsymbol{F}_{k, l}(n, m)+\boldsymbol{D} \boldsymbol{F}_{k, l}(n-1, m),, & (1 \leq n \leq k)(\mathrm{II}-3 a) \\
\boldsymbol{D F}_{k, l}(k, m), & (n=k+1)
\end{array}\right. \\
& \boldsymbol{F}_{k+1, l}(n, m)=\left\{\begin{array}{cc}
\boldsymbol{F}_{k, l}(n, 0)(\Theta \boldsymbol{I}+\boldsymbol{C}), & (m=0) \\
\boldsymbol{F}_{k, l}(n, m)(\Theta \boldsymbol{I}+\boldsymbol{C})+\boldsymbol{F}_{k, l}(n, m-1) \boldsymbol{D}, & (1 \leq m \leq l) \\
\boldsymbol{F}_{k, l}(n, l) \boldsymbol{D}, & (m=l+1)
\end{array}\right.
\end{aligned}
$$

where the matrix $\boldsymbol{F}_{k, l}(n, m)$ satisfies

$$
\left(\Theta \boldsymbol{I}+\boldsymbol{C}+z_{1} \boldsymbol{D}\right)^{n} \frac{\boldsymbol{D}}{\lambda}\left(\Theta \boldsymbol{I}+\boldsymbol{C}+z_{2} \boldsymbol{D}\right)^{m}=\sum_{k=0}^{n} \sum_{l=0}^{m} z_{1}^{k} z_{2}^{l} \boldsymbol{F}_{k, l}(n, m)
$$

with $\boldsymbol{F}_{0,0}(0,0)=\frac{D}{\lambda}$.

Using (II-3c) in (II-2), we get

$$
\begin{aligned}
\boldsymbol{\Omega}^{*}\left(z_{1}, z_{2}, \theta\right) & =\sum_{k=0}^{\infty} \sum_{l=0}^{\infty} z_{1}^{k} z_{2}^{l} \int_{x=0}^{\infty} \int_{t=0}^{x} e^{\theta t} e^{-(\Theta+\theta) x} \cdot \frac{v(x)}{E(V)} \\
& \times \sum_{n=k}^{\infty} \sum_{m=l}^{\infty} \frac{t^{n}(x-t)^{m}}{n ! m !} \boldsymbol{F}_{n, m}(k, l) d t d x .
\end{aligned}
$$

Then, we obtain the coefficient matrix $\boldsymbol{\Omega}_{k, l}^{*}(\theta)=\sum_{k=0}^{\infty} e^{-\theta y} d \boldsymbol{\Omega}_{k l}(y)$ of $\boldsymbol{\Omega}^{*}\left(z_{1}, z_{2}, \theta\right)$ as 
$\boldsymbol{\Omega}_{k, l}^{*}(\theta)=\int_{x=0}^{\infty} \int_{t=0}^{x} e^{\theta t} e^{-(\Theta+\theta) x} \frac{v(x)}{E(V)} \sum_{n=k}^{\infty} \sum_{m=l}^{\infty} \frac{t^{n}(x-t)^{m}}{n ! m !} \boldsymbol{F}_{n, m}(k, l) d t d x$.

Setting $\theta=0$, we get

$$
\boldsymbol{\Omega}_{k, l}=\sum_{n=k}^{\infty} \sum_{m=l}^{\infty} \gamma_{n, m} \boldsymbol{F}_{n, m}(k, l)
$$

where

$$
\gamma_{n, m}=\frac{1}{E(V)(n+m+1) !} \int_{0}^{\infty} x^{n+m+1} e^{-\Theta x} v(x) d x
$$

\section{Appendix III Proof of Theorem 3}

From (2.36) and after using $(\boldsymbol{C}+\boldsymbol{D} \boldsymbol{G}) \boldsymbol{e}=\mathbf{0}, \boldsymbol{H}(\boldsymbol{G}) \boldsymbol{e}=\boldsymbol{e}$ and $\left.\frac{d}{d z} \boldsymbol{G}(z)\right|_{z=1} \boldsymbol{e}=\boldsymbol{\mu}$, we have

$$
\begin{gathered}
\boldsymbol{\kappa}^{*}=\left.\frac{d}{d z} \boldsymbol{K}(z)\right|_{z=1} \boldsymbol{e} \\
=\left[\sum_{n=0}^{N-1} \boldsymbol{\Phi}_{n}(-\boldsymbol{C})^{-1} \boldsymbol{G}^{n} \boldsymbol{D} \boldsymbol{\mu}+\left.\frac{d}{d z} \boldsymbol{V}(\boldsymbol{G}(z))\right|_{z=1} \boldsymbol{e}\right] \\
+\left.\left[\sum_{n=0}^{N-1} \boldsymbol{\Phi}_{n}(-\boldsymbol{C})^{-1} \boldsymbol{G}^{n}(\boldsymbol{C}+\boldsymbol{D} \boldsymbol{G})+\boldsymbol{V}(\boldsymbol{G})\right] \frac{d}{d z} \boldsymbol{H}(\boldsymbol{G}(z))\right|_{z=1} \boldsymbol{e}
\end{gathered}
$$

( $\boldsymbol{\mu}$ is given by $\boldsymbol{\mu}=\left.\frac{d}{d z} \boldsymbol{G}(z)\right|_{z=1} \boldsymbol{e}=(\boldsymbol{I}-\boldsymbol{G}+\boldsymbol{e g})[\boldsymbol{I}-\boldsymbol{A}+(\boldsymbol{e}-\boldsymbol{\beta}) \boldsymbol{g}]^{-1} \boldsymbol{e}$, where $\boldsymbol{\beta}$ is the mean number of customers that arrive during a service time and is given by (see Neuts [12])

$$
\left.\boldsymbol{\beta}=\left.\frac{d}{d z} \boldsymbol{A}(z)\right|_{z=1} \boldsymbol{e}=\rho \boldsymbol{e}+(\boldsymbol{e} \boldsymbol{\pi}+\boldsymbol{C}+\boldsymbol{D})^{-1}(\boldsymbol{A}-\boldsymbol{I}) \boldsymbol{D} \boldsymbol{e}\right) .
$$

Using

$$
\frac{d}{d z} e^{(\boldsymbol{C}+\boldsymbol{D} \boldsymbol{G}(z) x}=\sum_{n=1}^{\infty} \frac{x^{n}}{n !} \sum_{k=0}^{n-1}(\boldsymbol{C}+\boldsymbol{D} \boldsymbol{G}(z))^{k} \boldsymbol{D}\left[\frac{d}{d z} \boldsymbol{G}(z)\right](\boldsymbol{C}+\boldsymbol{D} \boldsymbol{G}(z))^{n-1-k}
$$

and

$$
(\boldsymbol{C}+\boldsymbol{D} \boldsymbol{G})^{n-k 1} \boldsymbol{e}=\mathbf{0},(k \leq n-2),
$$

we get

$$
\left.\frac{d}{d z} e^{(\boldsymbol{C}+\boldsymbol{D} \boldsymbol{G}(z)) x}\right|_{z=1} \boldsymbol{e}=\sum_{n=1}^{\infty} \frac{x^{n}}{n !}(\boldsymbol{C}+\boldsymbol{D} \boldsymbol{G})^{n-1} \boldsymbol{D} \boldsymbol{\mu}
$$


Thus, we get

$$
\left.\frac{d}{d z} \boldsymbol{V}(\boldsymbol{G}(z))\right|_{z=1} \boldsymbol{e}=\int_{0}^{\infty} \sum_{n=1}^{\infty} \frac{x^{n}}{n !}(\boldsymbol{C}+\boldsymbol{D} \boldsymbol{G})^{n-1} \boldsymbol{D} \boldsymbol{\mu} d V(x)
$$

Multiplying (III-2) by $\boldsymbol{I}=(\boldsymbol{e g}+\boldsymbol{C}+\boldsymbol{D} \boldsymbol{G})^{-1}(\boldsymbol{e g}+\boldsymbol{C}+\boldsymbol{D} \boldsymbol{G}) \quad$ and $\quad$ using $\boldsymbol{g}(\boldsymbol{C}+\boldsymbol{D} \boldsymbol{G})^{n-1}=\mathbf{0},(n \geq 2)$, we get

$$
\left.\frac{d}{d z} \boldsymbol{V}(\boldsymbol{G}(z))\right|_{z=1} \boldsymbol{e}
$$

$$
=(\boldsymbol{e} \boldsymbol{g}+\boldsymbol{C}+\boldsymbol{D} \boldsymbol{G})^{-1} \int_{0}^{\infty}\left[x \boldsymbol{e} \boldsymbol{D} \boldsymbol{\mu}+\left(e^{(\boldsymbol{C}+\boldsymbol{D} \boldsymbol{G}) x}-\boldsymbol{I}\right) \boldsymbol{D} \boldsymbol{\mu}\right] d V(x) .
$$

Using $\boldsymbol{g} \boldsymbol{D} \boldsymbol{\mu}=\lambda /(1-\rho)$ (Lucantoni et al. [10]) and $\int_{0}^{\infty} e^{(\boldsymbol{C}+\boldsymbol{D} \boldsymbol{G}) x} d V(x)=\boldsymbol{V}(\boldsymbol{G})$, we get

$$
\left.\frac{d}{d z} \boldsymbol{V}(\boldsymbol{G}(z))\right|_{z=1} \boldsymbol{e}=\frac{\lambda E(V)}{1-\rho} \boldsymbol{e}+[\boldsymbol{V}(\boldsymbol{G})-\boldsymbol{I}](\boldsymbol{e g}+\boldsymbol{C}+\boldsymbol{D} \boldsymbol{G})^{-1} \boldsymbol{D} \boldsymbol{\mu}
$$

where we used $(\boldsymbol{e g}+\boldsymbol{C}+\boldsymbol{D G}) \boldsymbol{e}=\boldsymbol{e}$ and $(\boldsymbol{e g}+\boldsymbol{C}+\boldsymbol{D G})^{-1} \boldsymbol{e}=\boldsymbol{e}$.

Similarly, we get

$$
\left.\frac{d}{d z} \boldsymbol{H}(\boldsymbol{G}(z))\right|_{z=1} \boldsymbol{e}=\frac{\lambda E(H)}{1-\rho} \boldsymbol{e}+[\boldsymbol{H}(\boldsymbol{G})-\boldsymbol{I}](\boldsymbol{e} \boldsymbol{g}+\boldsymbol{C}+\boldsymbol{D} \boldsymbol{G})^{-1} \boldsymbol{D} \boldsymbol{\mu}
$$

Using (III-4) and (III-5) in (III-1) with $\boldsymbol{g}(\boldsymbol{e} \boldsymbol{g}+\boldsymbol{C}+\boldsymbol{D} \boldsymbol{G})^{-1}=\boldsymbol{g}$ and $\boldsymbol{G}^{n} \boldsymbol{e}=\boldsymbol{e}$ completes the proof. 


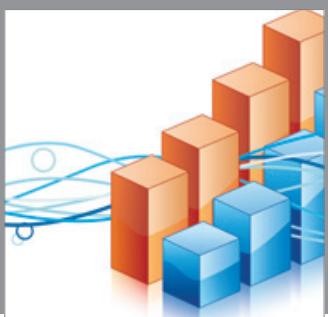

Advances in

Operations Research

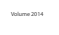

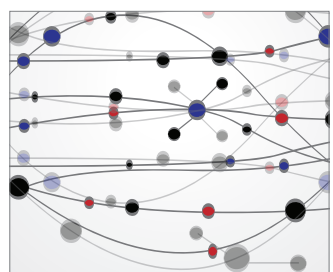

\section{The Scientific} World Journal
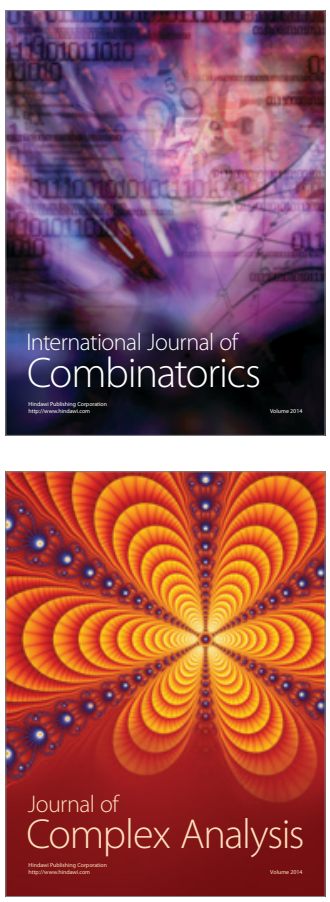

International Journal of

Mathematics and

Mathematical

Sciences
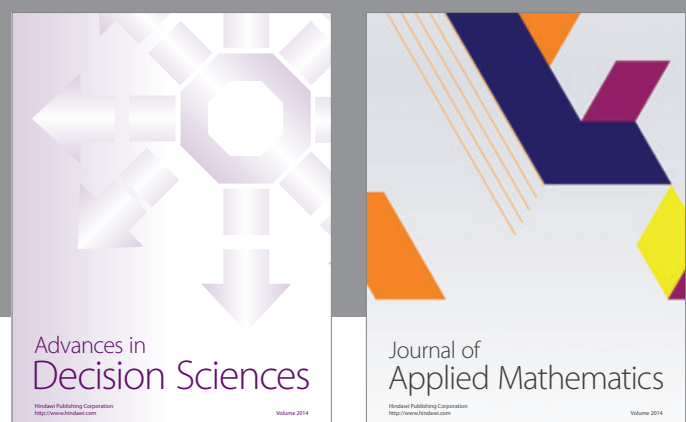

Journal of

Applied Mathematics
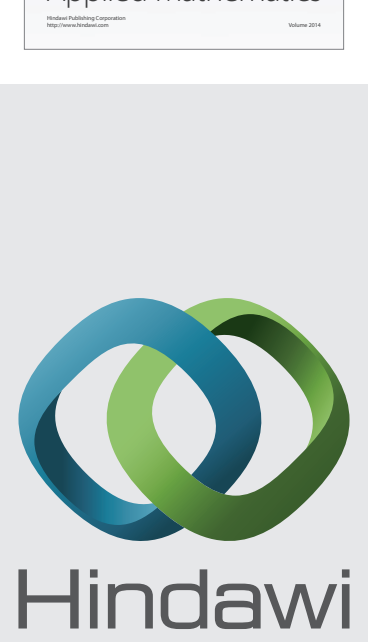

Submit your manuscripts at http://www.hindawi.com
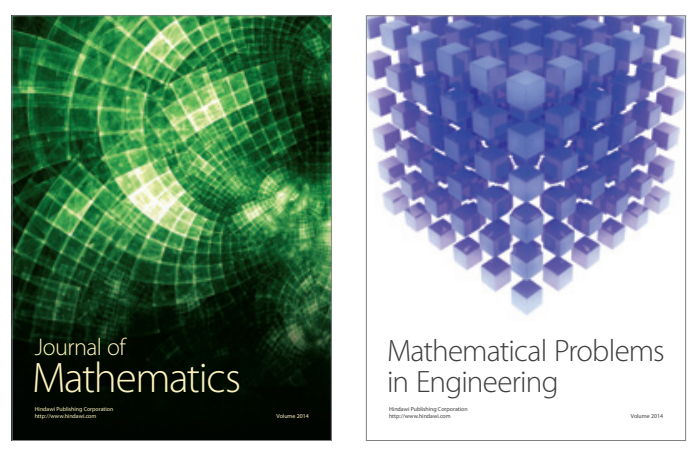

Mathematical Problems in Engineering
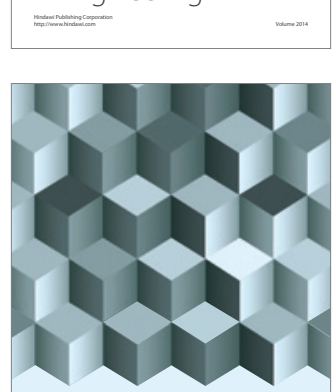

Journal of

Function Spaces
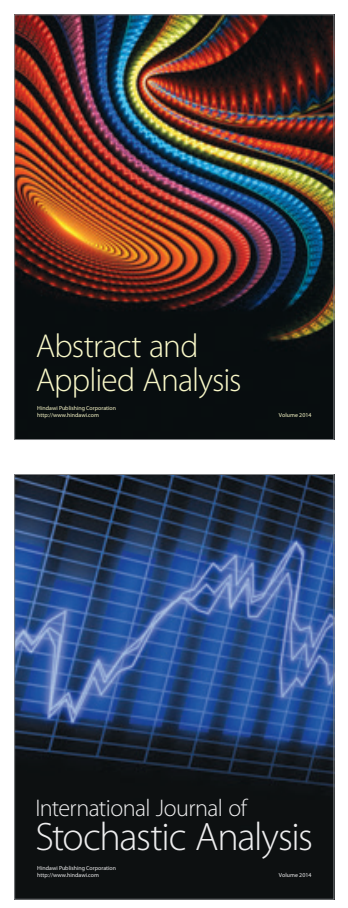

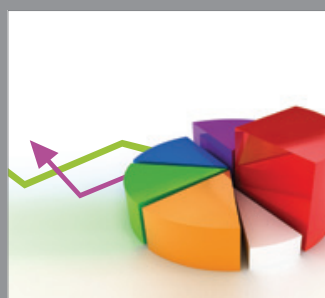

ournal of

Probability and Statistics

Promensencen
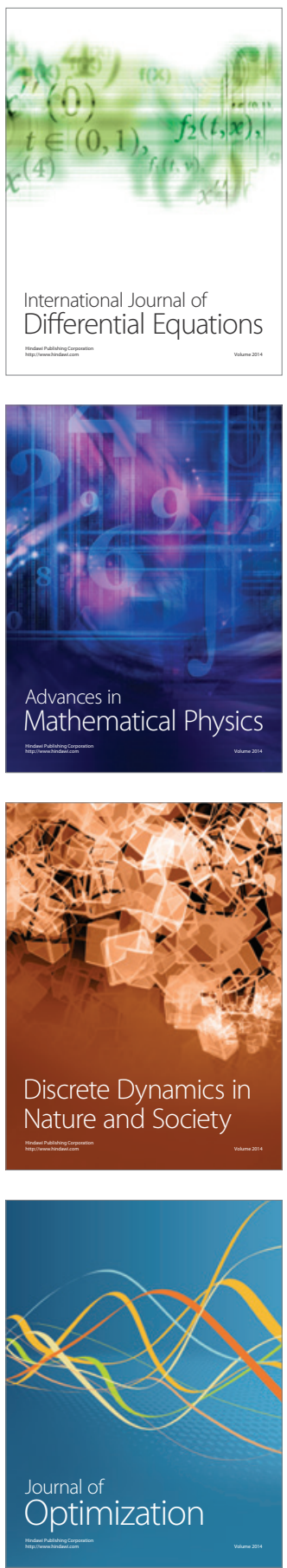